\title{
Operations on Locally Free Classgroups
}

\author{
by
}

\author{
BERNHARD KÖCK
}

\begin{abstract}
Let $\Gamma$ be a finite group and $K$ a number field. We show that the operation $\psi_{k}$ defined by Cassou-Noguès and Taylor on the locally free classgroup $\operatorname{Cl}\left(\mathcal{O}_{K} \Gamma\right)$ is a symmetric power operation if $\operatorname{gcd}(k, \operatorname{ord}(\Gamma))=1$. Using the equivariant Adams-Riemann-Roch theorem, we furthermore give a geometric interpretation of a formula established by Burns and Chinburg for these operations.
\end{abstract}

\section{Introduction}

Exterior or symmetric powers of modules play an important role in algebraic $K$-theory, in representation theory of finite groups, in number theory, or in algebraic geometry. The object of this paper is to work out their central role in a fascinating interplay of these four subjects described subsequently.

The $k$-th Adams operation $\psi^{k}$ on the classical ring $K_{0}(\mathbb{C} \Gamma)$ of virtual characters of a finite group $\Gamma$ can be described in the following two ways: In terms of modules, $\psi^{k}$ is given by

$$
\psi^{k}(M)=N_{k}\left(\Lambda_{\mathbb{C}}^{1}(M), \ldots, \Lambda_{\mathbb{C}}^{k}(M)\right) ;
$$

i. e. the exterior powers $\Lambda_{\mathbb{C}}^{1}(M), \ldots, \Lambda_{\mathbb{C}}^{k}(M)$ of the representation $M$ of $\Gamma$ are plugged into the $k$-th Newton polynomial $N_{k}=t_{1}^{k}+\cdots+t_{k}^{k}$ considered as a polynomial in the elementary symmetric functions of $t_{1}, \ldots, t_{k}$. In terms of characters, $\psi^{k}$ is given by

$$
\psi^{k}(\chi)(\gamma)=\chi\left(\gamma^{k}\right)
$$

(for $\gamma \in \Gamma$ and $\chi \in K_{0}(\mathbb{C} \Gamma)$ a character).

Now let $K$ be a number field. Let $K_{0}\left(\mathcal{O}_{K} \Gamma\right)$ denote the Grothendieck group of all projective modules over the group ring $\mathcal{O}_{K} \Gamma$ and let

$$
\mathrm{Cl}\left(\mathcal{O}_{K} \Gamma\right)=\operatorname{ker}\left(\operatorname{rank}: K_{0}\left(\mathcal{O}_{K} \Gamma\right) \rightarrow \mathbb{Z}\right)
$$


denote the locally free classgroup associated with $K$ and $\Gamma$. Fröhlich has established an isomorphism between $\mathrm{Cl}\left(\mathcal{O}_{K} \Gamma\right)$ and a certain residue class group of the group

$$
\operatorname{Hom}_{\mathrm{Gal}(\bar{K} / K)}\left(K_{0}(\bar{K} \Gamma), \mathcal{I}(\bar{K})\right)
$$

consisting of Galois-invariant homomorphisms from $K_{0}(\bar{K} \Gamma)=K_{0}(\mathbb{C} \Gamma)$ to the group $\mathcal{I}(\bar{K})$ of ideles of $\bar{K}$ (e. g. see Theorem 1 on p. 20 in [F1]). Using Taylor's group logarithm techniques, Cassou-Noguès and Taylor have shown that, under certain additional assumptions, the endomorphism $\operatorname{Hom}\left(\psi^{k}, \mathcal{I}(\bar{K})\right)$ of $\operatorname{Hom}_{\mathrm{Gal}(\bar{K} / K)}\left(K_{0}(\bar{K} \Gamma), \mathcal{I}(\bar{K})\right)$ induces an endomorphism on $\mathrm{Cl}\left(\mathcal{O}_{K} \Gamma\right)$ (e. g. see Chapter 9 in $\left.[\mathrm{T}]\right)$. This endomorphism will be denoted by $\psi_{k}^{\mathrm{CNT}}$ in the subsequent considerations. In the paper [BC], Burns and Chinburg have studied the question whether there is an algebraic description of $\psi_{k}^{\mathrm{CNT}}$, for instance in terms of power operations on $\mathcal{O}_{K} \Gamma$-modules. Their main result essentially is a formula for $\psi_{k}^{\mathrm{CNT}}(\mathfrak{A})$ where $\mathfrak{A}$ is an arbitrary $\Gamma$-stable fractional ideal in a tame Galois extension $N / K$ with Galois group $\Gamma$. Their formula expresses $\psi_{k}^{\mathrm{CNT}}(\mathfrak{A})$ in terms of powers of the different $\mathfrak{D}_{N / K}$ and of powers of the ideal $\mathfrak{A}$ (see Corollary 2.4 in $[\mathrm{BC}]$ ).

The central result of this paper is the identification of the endomorphism $\psi_{k}^{\mathrm{CNT}}$ with a symmetric power operation on $K_{0}\left(\mathcal{O}_{K} \Gamma\right)$ in the case $\operatorname{gcd}(k, \operatorname{ord}(\Gamma))=1$. The aim of this paper finally is to give a geometric interpretation of the formula of Burns and Chinburg, namely as an equivariant Adams-Riemann-Roch formula for the $\Gamma$-morphism $\operatorname{Spec}\left(\mathcal{O}_{N}\right) \rightarrow \operatorname{Spec}\left(\mathcal{O}_{K}\right)$.

The following fact (see Proposition 1.1) is the fundamental observation for our central result: Let $R$ be a commutative ring and $M$ a projective module over the group ring $R \Gamma$; if $\operatorname{gcd}(k, \operatorname{ord}(\Gamma))$ is invertible in $R$, then the $k$-th exterior power $\Lambda_{R}^{k}(M)$ and the $k$-th symmetric power $\operatorname{Sym}_{R}^{k}(M)$ are projective over $R \Gamma$ again; here, as usual, $\Gamma$ acts on the exterior or symmetric power diagonally.

A more general form of this observation will enable us to define a $k$-th symmetric power operation $\sigma^{k}$ on the Grothendieck group $K_{0}(R \Gamma)$ and, more generally, on Quillen's higher $K$-groups $K_{q}(R \Gamma), q \geq 0$, (see section 1 ). An obvious generalization of the moduletheoretic definition of $\psi^{k}$ on $K_{0}(\mathbb{C} \Gamma)$ explained above furthermore yields an (additive) $k$-th Adams operation $\psi^{k}$ on $K_{q}(R \Gamma), q \geq 0$, if $\operatorname{gcd}(k, \operatorname{ord}(\Gamma))$ is invertible in $R$ (see section 1). In Theorem 1.6, we will prove several standard properties of these operations such as e. g. the compatibility with the Cartan homomorphism. Moreover, Theorem 1.6 contains a proof of the identity

$$
\psi^{k}([R \Gamma])=[R \Gamma]
$$

conjectured in (1.12) in Chapter 9 of $[\mathrm{T}]$.

In section 2 , we will show that $\psi^{k}$ is multiplicative. Since there is no splitting principle available, this can not be proved as e. g. in [FL]. Instead, we will use the universal form of the Cauchy decomposition and of the Pieri formula (established by Akin, Buchsbaum and Weyman in characteristic-free representation theory) in order to express the exterior power $\Lambda^{i}(M \otimes N)$ of the tensor product of two modules as a universal polynomial in the exterior powers of $M$ and $N$. There remains open the problem how to prove that $\psi^{k} \circ \psi^{l}=\psi^{k l}$ on $K_{0}(R \Gamma)$ (here, $l$ denotes another natural number such that $\operatorname{gcd}(l, \operatorname{ord}(\Gamma))$ is invertible in $R$ ). 
We now assume that $\operatorname{gcd}(k, \operatorname{ord}(\Gamma))=1$ and fix a $k^{\prime} \in \mathbb{N}$ with $k k^{\prime} \equiv 1 \bmod \operatorname{ord}(\Gamma)$. By the constructions explained above, we have operations $\sigma^{k}$ and $\psi^{k}$ on $K_{0}\left(\mathcal{O}_{K} \Gamma\right)$ and, in particular, on $\mathrm{Cl}\left(\mathcal{O}_{K} \Gamma\right)$ by restricting. The precise form of our central result now is:

$$
\psi_{k^{\prime}}^{\mathrm{CNT}}=\sigma^{k} \quad \text { and } \quad k \cdot \psi_{k^{\prime}}^{\mathrm{CNT}}=\psi^{k} \quad \text { on } \quad \mathrm{Cl}\left(\mathcal{O}_{K} \Gamma\right)
$$

(see Theorem 3.7). This result in particular answers the question (raised in (1.12) in Chapter 9 of $[\mathrm{T}]$ ) how the endomorphism $\psi_{k}^{\mathrm{CNT}}$ behaves with respect to the Cartan homomorphism (see Corollary 3.9).

Now let $N / K$ be a tame Galois extension with Galois group $\Gamma$. In section 4, we will essentially establish the following simple representation of the cotangential element $T_{f}^{\vee}$ associated with the $\Gamma$-morphism $f: \operatorname{Spec}\left(\mathcal{O}_{N}\right) \rightarrow \operatorname{Spec}\left(\mathcal{O}_{K}\right)$ :

$$
T_{f}^{\vee}=\left[\mathfrak{D}_{N / K}^{-1}\right]-\left[\mathcal{O}_{N}\right]
$$

Here, as above, $\mathfrak{D}_{N / K}$ denotes the different associated with $N / K$.

This representation of $T_{f}^{\vee}$ implies a particularly simple shape of the Bott multiplier and then of the equivariant Adams-Riemann-Roch formula for $f$ (see Theorem 5.4). On the other hand, we will reformulate the formula of Burns and Chinburg mentioned above using the central result Theorem 3.7 (see Theorem 5.6). It will then turn out that, more or less, the formula of Burns and Chinburg is a strengthening of the equivariant AdamsRiemann-Roch formula.

Acknowledgements. First of all, I would like to thank C. Greither for inviting me to stay with him at Université Laval in Québec for a few weeks in September/October 1996. In discussions during this visit, he has made the observation Proposition 1.1 which has later turned out to be the most important tool in understanding the operations on locally free classgroups defined by Cassou-Noguès and Taylor. Moreover, he has contributed several algebraic arguments which have considerably improved the paper. It is a pleasure to me to thank him for all this assistance. Furthermore, I would like to thank J. Weyman for the reference to the universal form of the Pieri formula and for long e-mail discussions about the plethysm problem. Finally, I would like to thank D. Burns who asked me some time ago how to geometrically interpretate his formula developed jointly with T. Chinburg.

Notations. Let $\Gamma$ be a finite group and let $R$ be a commutative $\Gamma$-ring, i. e. a commutative ring together with an action of $\Gamma$ by ring automorphisms. The twisted group ring associated with $\Gamma$ and $R$ is denoted by $R \# \Gamma$. If $\Gamma$ acts on $R$ trivially, we simply write $R \Gamma$ for $R \# \Gamma$. We denote the Grothendieck group of all finitely generated projective $R \# \Gamma$-modules (of all finitely generated $R$-projective $R \# \Gamma$-modules, of all finitely generated $R \# \Gamma$-modules) by $K_{0}(R \# \Gamma)$, (by $K_{0}(\Gamma, R)$, and by $K_{0}^{\prime}(R \# \Gamma)$, respectively). We have a canonical homomorphism $c: K_{0}(R \# \Gamma) \rightarrow K_{0}(\Gamma, R)$ which is called the Cartan homomorphism. If the order of the group $\Gamma$ is invertible in $R, c$ is bijective (e. g. see the proof of Corollary 2.2c) in [Ko4]) and $K_{0}(R \# \Gamma)$ will be identified with $K_{0}(\Gamma, R)$. Furthermore, we have a canonical homomorphism $K_{0}(\Gamma, R) \rightarrow K_{0}^{\prime}(R \# \Gamma)$. If $R$ is regular (e. g. if $R$ is a field or a ring of integers in a number field), this homomorphism is bijective (e. g. see $\operatorname{Satz}(2.1)$ in [Ko1]) and $K_{0}(\Gamma, R)$ will be identified with $K_{0}^{\prime}(R \# \Gamma)$. Thus, if $R$ is 
a field of characteristic zero, all three Grothendieck groups coincide. The corresponding higher $K$-groups will be denoted by $K_{q}(R \# \Gamma), K_{q}(\Gamma, R), K_{q}^{\prime}(R \# \Gamma), q \geq 0$, (see [Q]). The corresponding identifications hold for higher $K$-groups as well. For $K_{1}(R \# \Gamma)$, we will use also the following two descriptions (e. g. see [B]): By the third theorem on p. 228 in $[\mathrm{Gr} 1], K_{1}(R \# \Gamma)$ is isomorphic to the abelianized infinite linear group $\mathrm{GL}_{\infty}(R \# \Gamma)^{\mathrm{ab}}$; furthermore, $K_{1}(R \# \Gamma)$ is the free abelian group over all pairs $(M, \alpha)$, where $M$ is a $\mathrm{f}$. $\mathrm{g}$. projective $R \# \Gamma$-module and $\alpha$ is an $R \# \Gamma$-automorphism of $M$, modulo the relations defined on p. 348 in [B]. The notation $K_{0} T(-)$ will always stand for a Grothendieck group of torsion modules.

\section{$\S 1$ Power Operations for Projective Modules over Group Rings}

Let $\Gamma$ be a finite group, $R$ a commutative $\Gamma$-ring and $k$ a natural number such that $\operatorname{gcd}(k, \operatorname{ord}(\Gamma))$ is invertible in $R$.

The aim of this section is to construct a symmetric power operation $\sigma^{k}$, an exterior power operation $\lambda^{k}$, and an Adams operation $\psi^{k}$ on Quillen's higher $K$-groups $K_{q}(R \# \Gamma), q \geq 0$, in particular on the Grothendieck group $K_{0}(R \# \Gamma)$ of all f. g. projective $R \# \Gamma$-modules. Furthermore, we will prove several standard properties of these operations and we will show the equality $\psi^{k}([R \# \Gamma])=[R \# \Gamma]$ if $\operatorname{gcd}(k, \operatorname{ord}(\Gamma))=1$. The following proposition is the fundamental observation for this equality and for the whole section.

Proposition 1.1. Let $k_{1}, \ldots, k_{r} \in \mathbb{N}$ with $k_{1}+\cdots+k_{r}=k$ and let $M_{1}, \ldots, M_{r}$ be projective $R \# \Gamma$-modules. Then the $R \# \Gamma$-module

$$
P:=\operatorname{Sym}^{k_{1}}\left(M_{1}\right) \otimes \cdots \otimes \operatorname{Sym}^{k_{r}}\left(M_{r}\right)
$$

is projective over $R \# \Gamma$, too. (Here, all tensor products and all symmetric powers in $P$ are formed over $R$ and $\Gamma$ acts on $P$ diagonally.)

If even $\operatorname{gcd}(k, \operatorname{ord}(\Gamma))=1$ and $M_{1}, \ldots, M_{r}$ are free over $R \# \Gamma$, then $P$ is free over $R \# \Gamma$, too.

Proof. For a moment, let $k \in \mathbb{N}$ be arbitrary and let $M$ be a free $R \# \Gamma$-module. Let $B \subset M$ be an $R$-basis of $M$ which carries a free action of $\Gamma$. For instance, the basis consisting of the $\Gamma$-images of an $R \# \Gamma$-basis of $M$ is such a basis. Let $B^{k} / \Sigma_{k}$ denote the set of unordered $k$-tuples in $B$. For any $H=\left(b_{1}, \ldots, b_{k}\right) \in B^{k} / \Sigma_{k}$, let $b_{H}:=\prod_{j=1}^{k} b_{j}$ denote the corresponding standard basis element of $\operatorname{Sym}^{k}(M)$. Then we obviously have $\gamma\left(b_{H}\right)=b_{\gamma(H)}$ for all $\gamma \in \Gamma$, where the action of $\Gamma$ on $B^{k} / \Sigma_{k}$ is defined in the obvious way. For any $H \in B^{k} / \Sigma_{k}$, the stabilizer $\operatorname{Stab}(H):=\{\gamma \in \Gamma: \gamma(H)=H\}$ clearly acts on the sets $H(n):=\{b \in B: b$ occurs $n$ times in $H\}, n \geq 1$. Thus, the order $\operatorname{ord}(\operatorname{Stab}(H))$ divides the number $\# H(n)$ of elements in $H(n)$ for all $n$. Hence, it divides $k=\sum_{n=1}^{\infty} n \cdot(\# H(n))$, too.

Now let $k \in \mathbb{N}$ such that $\operatorname{gcd}(k, \operatorname{ord}(\Gamma))$ is invertible in $R, k_{1}, \ldots, k_{r} \in \mathbb{N}$ with $k_{1}+\cdots+k_{r}=$ $k$, and $M_{1}, \ldots, M_{r}$ free $R \# \Gamma$-modules. Furthermore, let $B_{1}, \ldots, B_{r}$ be bases of $M_{1}, \ldots, M_{r}$ 
as above. Then, for any $H=\left(H^{1}, \ldots, H^{r}\right) \in B_{1}^{k_{1}} / \Sigma_{k_{1}} \times \cdots \times B_{r}^{k_{r}} / \Sigma_{k_{r}}$, the element $b_{H}:=b_{H^{1}} \otimes \cdots \otimes b_{H^{r}}$ is a standard basis element of $P$ with $\gamma\left(b_{H}\right)=b_{\gamma(H)}$. The order $\operatorname{ord}(\operatorname{Stab}(H))$ of the stabilizer of $H$ divides the order of the stabilizer of $H^{i}$ for all $i$. Then, by the first part of the proof, it divides $k=\sum_{i=1}^{r} k_{i}$, too. Hence, $\operatorname{ord}(\operatorname{Stab}(H))$ is invertible in $R$. Thus, the canonical $R \# \Gamma$-linear surjection $R \# \Gamma \rightarrow R \#[\Gamma / \operatorname{Stab}(H)]$, $\gamma \mapsto[\gamma \operatorname{Stab}(H)]$, can be split by the well-defined $R \# \Gamma$-linear map

$$
R \#[\Gamma / \operatorname{Stab}(H)] \rightarrow R \# \Gamma, \quad[\gamma \operatorname{Stab}(H)] \mapsto \frac{1}{\operatorname{ord}(\operatorname{Stab}(H))} \sum_{\gamma^{\prime} \in \operatorname{Stab}(H)}\left[\gamma \gamma^{\prime}\right] .
$$

This proves that the $R \# \Gamma$-module $R \#[\Gamma / \operatorname{Stab}(H)]$ is projective over $R \# \Gamma$. The $R \# \Gamma$ submodule generated by $b_{H}$ obviously is isomorphic to $R \#[\Gamma / \operatorname{Stab}(H)]$. Thus, it is $R \# \Gamma$ projective, too. Being a direct sum of such modules, also $P$ is projective. This shows the first assertion in Proposition 1.1 for free $R \# \Gamma$-modules $M_{1}, \ldots, M_{r}$ and, by passing to direct summands, also for projective $R \# \Gamma$-modules.

If finally $\operatorname{gcd}(k, \operatorname{ord}(\Gamma))=1$, then $\operatorname{Stab}(H)$ is trivial. Hence, $P$ is a free $R \# \Gamma$-module.

Now we are going to construct the power operations mentioned above. For this, it is convenient to define the following exact subcategories $\mathcal{M}_{i}, i=1,2, \ldots$ of the exact category $\mathcal{M}$ of all f. g. $R$-projective $R \# \Gamma$-modules: Let $\mathcal{M}_{i}$ be the smallest full subcategory of $\mathcal{M}$ which is closed under extensions and kernels of $R \# \Gamma$-epimorphisms and which contains all modules of the form $\operatorname{Sym}^{k_{1}}\left(M_{1}\right) \otimes \cdots \otimes \operatorname{Sym}^{k_{r}}\left(M_{r}\right)$, where $M_{1}, \ldots, M_{r}$ are f. g. projective $R \# \Gamma$-modules and $k_{1}, \ldots, k_{r}$ are natural numbers with $k_{1}+\cdots+k_{r}=i$. We call the modules in $\mathcal{M}_{i}$ modules of weight $i$. Obviously, $\mathcal{M}_{1}$ is the category of all $\mathrm{f}$. g. projective $R \# \Gamma$-modules. By Proposition 1.1, the category $\mathcal{M}_{k}$ is contained in $\mathcal{M}_{1}$.

Lemma 1.2. For all $i, j \in \mathbb{N}$, the tensor product induces a functor

$$
\otimes: \mathcal{M}_{i} \times \mathcal{M}_{j} \rightarrow \mathcal{M}_{i+j}
$$

Proof. A module of weight $i$ is called of level 0 iff it is of the form $\operatorname{Sym}^{k_{1}}\left(M_{1}\right) \otimes \cdots \otimes$ $\operatorname{Sym}^{k_{r}}\left(M_{r}\right)$ as above. Inductively, a module of weight $i$ is called of level $\leq m$ iff it is an extension or the kernel of an $R \# \Gamma$-epimorphism of two modules of weight $i$ and level $\leq m-1$. Then one easily shows by induction that the tensor product of a module of weight $i$ and level $\leq m$ with a module of weight $j$ and level $\leq n$ is a module of weight $i+j$ and level $\leq m+n$. Since any module of weight $i$ or $j$ is of finite level, this shows Lemma 1.2.

For any exact category $\mathcal{P}$ and for any $i \geq 1$, let $\mathcal{F}_{i}(\mathcal{P})$ denote the category of all sequences $M_{1} \hookrightarrow \cdots \hookrightarrow M_{i}$ of admissible monomorphism in $\mathcal{P}$ of length $i$. Here, a morphism $M^{\prime} \rightarrow M$ in $\mathcal{P}$ is called admissible iff it can be completed to a short exact sequence $0 \rightarrow M^{\prime} \rightarrow M \rightarrow M^{\prime \prime} \rightarrow 0$ in $\mathcal{P}$.

Lemma 1.3. For any sequence $M_{1} \hookrightarrow \cdots \hookrightarrow M_{i}$ in $\mathcal{F}_{i}\left(\mathcal{M}_{1}\right)$, the $R \# \Gamma$-module

$$
M_{1} \cdots M_{i}:=\operatorname{Image}\left(M_{1} \otimes \cdots \otimes M_{i} \stackrel{\text { can }}{\longrightarrow} \operatorname{Sym}^{i}\left(M_{i}\right)\right)
$$

is contained in $\mathcal{M}_{i}$. 
Proof. In the canonical filtration

$$
M_{1} \cdots M_{i} \subseteq M_{1} \cdots M_{i-2} M_{i} M_{i} \subseteq \cdots \subseteq \operatorname{Sym}^{i}\left(M_{i}\right)
$$

of $\operatorname{Sym}^{i}\left(M_{i}\right)$, each successive quotient is isomorphic to one of the modules $M_{1} \cdots M_{r-1} \otimes$ $\operatorname{Sym}^{i-r+1}\left(\frac{M_{i}}{M_{r}}\right), r=1, \ldots, i$. Now, Lemma 1.3 follows from Lemma 1.2 by induction on $i$.

It is easy to prove that the exact categories $\mathcal{M}_{1}, \mathcal{M}_{2}, \ldots$ together with the tensor products $\otimes: \mathcal{M}_{i} \times \mathcal{M}_{j} \rightarrow \mathcal{M}_{i+j}, i, j \in \mathbb{N}$, (well-defined by Lemma 1.2) and the symmetric power operations $\mathcal{F}_{i}\left(\mathcal{M}_{1}\right) \rightarrow \mathcal{M}_{i},\left(M_{1} \hookrightarrow \cdots \hookrightarrow M_{i}\right) \mapsto M_{1} \cdots M_{i}$, (well-defined by Lemma 1.3) satisfy the axioms (E1) through (E5) in section 7 in [Gr3]. Unlike Grayson in section 7 in [Gr3], we can not define operations $\mathcal{F}_{i}\left(\mathcal{M}_{j}\right) \rightarrow \mathcal{M}_{i j}$ for $j>1$, since it is not clear whether the $i$-th symmetric power $\operatorname{Sym}^{i}(M)$ of a module $M$ of weight $j$ is a module of weight $i j$. This problem is related to the so-called plethysm problem (see also Remark 2.6). Nevertheless, we may apply the construction of section 7 in [Gr3] for $j=1$. It yields continuous maps

$$
\sigma^{i}:\left|G \mathcal{M}_{1}\right| \rightarrow\left|G^{i} \mathcal{M}_{i}\right|, \quad i \geq 1,
$$

from the geometric realization of the $G$-construction associated with $\mathcal{M}_{1}$ to the geometric realization of the $i$-fold iterated $G$-construction associated with $\mathcal{M}_{i}$. By passing to homotopy groups, we obtain symmetric power operations

$$
\sigma^{i}: K_{q}(R \# \Gamma) \rightarrow K_{q}\left(\mathcal{M}_{i}\right), \quad q \geq 0, \quad i \geq 1,
$$

on Quillen's $K$-groups. For $i=k$, we finally obtain a symmetric power operation

$$
\sigma^{k}: K_{q}(R \# \Gamma) \rightarrow K_{q}(R \# \Gamma)
$$

on the higher $K$-groups $K_{q}(R \# \Gamma), q \geq 0$, since $\mathcal{M}_{k}$ is contained in $\mathcal{M}_{1}$ by Proposition 1.1 .

Lemma 1.4. For any sequence $M_{1} \hookrightarrow \cdots \hookrightarrow M_{i}$ in $\mathcal{F}_{i}\left(\mathcal{M}_{1}\right)$, the $R \# \Gamma$-module

$$
M_{1} \wedge \ldots \wedge M_{i}:=\operatorname{Image}\left(M_{1} \otimes \cdots \otimes M_{i} \stackrel{\text { can }}{\longrightarrow} \Lambda^{i}\left(M_{i}\right)\right)
$$

is contained in $\mathcal{M}_{i}$.

Proof. For any $i \geq 1$ and any f. g. projective $R \# \Gamma$-module $M$, the Koszul complex

$$
0 \rightarrow \Lambda^{i}(M) \rightarrow M \otimes \Lambda^{i-1}(M) \rightarrow \cdots \rightarrow \operatorname{Sym}^{i-1}(M) \otimes M \rightarrow \operatorname{Sym}^{i}(M) \rightarrow 0
$$

is an exact sequence of $R \# \Gamma$-modules. It follows from Lemma 1.2 by induction on $i$ that $\Lambda^{i}(M)$ is contained in $\mathcal{M}_{i}$. Now, Lemma 1.4 can be deduced from this fact as in the proof of Lemma 1.3.

Remark 1.5. Lemma 1.4, Lemma 1.2, and Proposition 1.1 imply that Proposition 1.1 holds as well, if some symmetric powers are replaced by exterior powers in the definition of $P$. This can of course also be shown directly as in Proposition 1.1 by introducing some signs. Moreover, it is more convenient to define the categories $\mathcal{M}_{i}, i \geq 1$, with symmetric 
powers than with exterior powers since it would otherwise not be clear whether symmetric powers (and, more generally, (co)Schur modules, see Proposition 2.1) are contained in the categories $\mathcal{M}_{i}, i \geq 1$.

As above, we now obtain exterior power operations

$$
\lambda^{i}:\left|G \mathcal{M}_{1}\right| \rightarrow\left|G^{i} \mathcal{M}_{i}\right|, \quad i \geq 1,
$$

and the corresponding maps on the $K$-groups.

By Lemma 1.2, we furthermore have continuous maps

$$
\otimes:\left|G^{i} \mathcal{M}_{i}\right| \times\left|G^{j} \mathcal{M}_{j}\right| \rightarrow\left|G^{i+j} \mathcal{M}_{i+j}\right|, \quad i, j \geq 1,
$$

(see also section 1 of $[\mathrm{Ko} 2])$. Let $N_{i}\left(s_{1}, \ldots, s_{i}\right)$ denote the $i$-th Newton polynomial $t_{1}^{i}+$ $\cdots+t_{i}^{i}$ considered as a polynomial in the elementary symmetric functions $s_{1}, \ldots, s_{i}$ of $t_{1}, \ldots, t_{i}$. Replacing $s_{1}, \ldots, s_{i}$ by the exterior power operations $\lambda^{1}, \ldots, \lambda^{i}$ defined above and the products in $N_{i}\left(s_{1}, \ldots, s_{i}\right)$ by the tensor products defined above, we finally obtain continuous maps

$$
\psi^{i}:\left|G \mathcal{M}_{1}\right| \rightarrow\left|G^{i} \mathcal{M}_{i}\right|, \quad i \geq 1,
$$

and the corresponding maps on the $K$-groups as above. These maps are called Adams operations.

Applying Grayson's construction to the exact category $\mathcal{M}$ consisting of all $\mathrm{f}$. g. $R$ projective $R \# \Gamma$-modules (i. e. all categories $\mathcal{M}_{i}, i \geq 1$, in section 7 in [Gr3] are identical to $\mathcal{M})$, we similarly obtain power operations

$$
\sigma^{i}, \lambda^{i}, \psi^{i}: K_{q}(\Gamma, R) \rightarrow K_{q}(\Gamma, R), \quad i \geq 0, \quad q \geq 0,
$$

(see also $\S 2$ and $\S 3$ in $[\mathrm{Ko} 1]$ ).

\section{Theorem 1.6.}

(a) Let $\gamma$ be one of the operations $\sigma^{i}, \lambda^{i}, \psi^{i}, i \geq 1$. Then the following diagram commutes for all $q \geq 0$ :

$$
\begin{array}{ccc}
K_{q}(R \# \Gamma) & \stackrel{c}{\longrightarrow} & K_{q}(\Gamma, R) \\
\downarrow \gamma & & \downarrow \gamma \\
K_{q}\left(\mathcal{M}_{i}\right) & \stackrel{\text { can }}{\longrightarrow} & K_{q}(\Gamma, R) .
\end{array}
$$

In particular, the operations $\sigma^{k}, \lambda^{k}$, and $\psi^{k}$ on $K_{q}(R \# \Gamma)$ are compatible with the corresponding operations on $K_{q}(\Gamma, R)$ with respect to the Cartan homomorphism (for all $q \geq 0)$.

(b) The operations $\sigma^{i}, \lambda^{i}, \psi^{i}, i \geq 1$, commute with base change with respect to any homomorphism $R \rightarrow R^{\prime}$ of $\Gamma$-rings and they commute with the restriction map for any subgroup $\Gamma^{\prime}$ of $\Gamma$.

(c) For all $i \geq 1$ and for all $M, N \in \mathcal{M}_{1}$ we have in $K_{0}\left(\mathcal{M}_{i}\right)$ :

(i) $\sigma^{i}([M]-[N])=\sum_{\substack{a \geq 0, b_{1}, \ldots, b_{u} \geq 1 \\ a+b_{1}+\cdots+b_{u}=i}}(-1)^{u}\left[\operatorname{Sym}^{a}(M) \otimes \operatorname{Sym}^{b_{1}}(N) \otimes \cdots \otimes \operatorname{Sym}^{b_{u}}(N)\right]$.

(ii) The assertion (i) analogously holds for exterior powers. 
(iii) $\psi^{i}([M]+[N])=\psi^{i}([M])+\psi^{i}([N])$.

(iv) $\sum_{j=0}^{i}(-1)^{j} \lambda^{j}([M]) \cdot \sigma^{i-j}([M])=0$.

(d) For all $i \geq 1$ and $q \geq 1$, we have:

(i) The maps $\lambda^{i}, \sigma^{i}, \psi^{i}: K_{q}(R \# \Gamma) \rightarrow K_{q}\left(\mathcal{M}_{i}\right)$ are homomorphisms.

(ii) $\sigma^{i}=(-1)^{i-1} \lambda^{i}: K_{q}(R \# \Gamma) \rightarrow K_{q}\left(\mathcal{M}_{i}\right)$.

(iii) $\psi^{i}=(-1)^{i-1} i \lambda^{i}: K_{q}(R \# \Gamma) \rightarrow K_{q}\left(\mathcal{M}_{i}\right)$.

(e) If $\operatorname{gcd}(k, \operatorname{ord}(\Gamma))=1$, we have:

$$
\psi^{k}([R \# \Gamma])=[R \# \Gamma] \quad \text { in } \quad K_{0}(R \# \Gamma) .
$$

(f) Let $\tilde{K}_{0}(\mathbb{Z}, R \# \Gamma):=\operatorname{ker}\left(K_{0}(\mathbb{Z}, R \# \Gamma) \stackrel{\text { can }}{\longrightarrow} K_{0}(R \# \Gamma)\right)$ denote the reduced Grothendieck group of all pairs $(M, \alpha)$, where $M$ is a f. g. projective $R \# \Gamma$-module and $\alpha$ is an $R \# \Gamma$-automorphism of $M$. Let $\gamma: \tilde{K}_{0}(\mathbb{Z}, R \# \Gamma) \rightarrow \tilde{K}_{0}(\mathbb{Z}, R \# \Gamma)$ be one of the operations $\sigma^{k}, \lambda^{k}, \psi^{k}$ defined similarly as above. Then the following diagram commutes:

$$
\begin{array}{ccc}
\tilde{K}_{0}(\mathbb{Z}, R \# \Gamma) & \stackrel{\text { can }}{\longrightarrow} & K_{1}(R \# \Gamma) \\
\downarrow \gamma & & \downarrow \gamma \\
\tilde{K}_{0}(\mathbb{Z}, R \# \Gamma) & \stackrel{\text { can }}{\longrightarrow} & K_{1}(R \# \Gamma) .
\end{array}
$$

Proof. The assertions (a) and (b) follow from the functoriality of Grayson's construction. The assertions (c)(i) and (c)(ii) are proved in section 8 in [Gr3]. The $\lambda$-series $\lambda_{t}([M]):=$ $\sum_{i \geq 0} \lambda^{i}([M]) t^{i}$ satisfies the rule $\lambda_{t}([M+N])=\lambda_{t}([M]) \cdot \lambda_{t}([N])$ by (c)(ii); being the logarithmic derivative of the $\lambda$-series (see page 23 in [FL]), the $\psi$-series is additive. This proves assertion (c)(iii). The assertion (c)(iv) follows from the fact that the Koszul complex

$$
0 \rightarrow \Lambda^{i}(M) \rightarrow M \otimes \Lambda^{i-1}(M) \rightarrow \cdots \rightarrow \operatorname{Sym}^{i-1}(M) \otimes M \rightarrow \operatorname{Sym}^{i}(M) \rightarrow 0
$$

is an exact sequence of $R \# \Gamma$-modules.

The assertion $(d)(i)$ follows from the fact that maps on higher homotopy groups which are induced by continuous maps are homomorphisms. By a similar argument, the products $K_{q}\left(\mathcal{M}_{i}\right) \times K_{q}\left(\mathcal{M}_{j}\right) \rightarrow K_{q}\left(\mathcal{M}_{i+j}\right)$ induced by the tensor product $\mathcal{M}_{i} \times \mathcal{M}_{j} \rightarrow \mathcal{M}_{i+j}$ are trivial for $q \geq 1$. This together with Newton's recursion formula proves assertion (d)(iii). Assertion (d)(ii) can be proved as in Theorem 6.1 in [Ko2].

To show assertion (e), we express $\psi^{k}$ as a polynomial in the symmetric power operations $\sigma^{1}, \ldots, \sigma^{k}$ using (c)(iv). Then all monomials in this polynomial are of weight $k$. Thus, by Proposition 1.1, there is an $n \in \mathbb{N}$ such that $\psi^{k}([R \# \Gamma])=n \cdot[R \# \Gamma]$. We have $n=1$ since $\operatorname{rank}_{R}\left(\psi^{k}([R \# \Gamma])\right)=\operatorname{rank}_{R}([R \# \Gamma])$. This proves assertion (e).

The assertion (f) can be proved as in section 9 in [Gr3] (see also the proof of Theorem 3.3 in $[\mathrm{Ko} 4])$.

\section{Remark 1.7.}

(a) If even $k$ is invertible in $R$, we have constructed an Adams operation $\psi^{k}$ on $K_{q}(R \# \Gamma)$, $q \geq 0$, in [Ko5] by using so-called cyclic powers. One should be able to prove that that operation agrees with the Adams operation defined in this section. Since there is no splitting principle available for $K_{0}(R \# \Gamma)$, this is a problem already (and in fact mainly) 
for $K_{0}(R \# \Gamma)$. At the end of the paper [Ko5], some speculations are given from which a solution for this problem would follow. If $R$ is a field and $\Gamma$ acts on $R$ trivially, the Cartan homomorphism $c: K_{0}(R \Gamma) \rightarrow K_{0}(\Gamma, R)$ is injective (see $\S 5$ in [Ke]). Since both operations $\psi^{k}$ commute with $c$, they agree in this case. In particular, Proposition 1.1 yields a considerable simplification of $\S 5$ in $[\mathrm{Ke}]$.

(b) Compared with the construction of this section, the construction in [Ko5] has the disadvantage that it is only possible under the assumption $k \in R^{\times}$. However, it has the advantage that both the multiplicativity of $\psi^{k}$ and the rule $\psi^{k} \circ \psi^{l}=\psi^{l} \circ \psi^{k}$ can rather easily be proved and that the definition of $\psi^{k}([M])$ consists of only two modules, if $k$ is a prime number.

Remark 1.8. Let $\Gamma^{\prime}$ be a subgroup $\Gamma$. In $\S 6$ of [Ko3], the following question has been raised: Under which conditions does the equality $\psi^{k}\left(\left[R \#\left[\Gamma / \Gamma^{\prime}\right]\right]\right)=\left[R \#\left[\Gamma / \Gamma^{\prime}\right]\right]$ hold in $K_{0}(\Gamma, R)$. Using the equivariant Adams-Riemann-Roch formula, we have proved in $\S 6$ in [Ko3] that this equality holds in a certain completion of $K_{0}(\Gamma, R)\left[k^{-1}\right]$ for all $k \in \mathbb{N}$. By Theroem 1.6(e), we now have that this equality holds already in $K_{0}(\Gamma, R)$ if $\Gamma^{\prime}$ is a normal subgroup and $\operatorname{gcd}\left(k, \operatorname{ord}\left(\Gamma / \Gamma^{\prime}\right)\right)=1$.

\section{$\S 2$ Multiplicativity of Adams Operations}

Let $\Gamma$ be a finite group, $R$ a commutative $\Gamma$-ring, and $k \in \mathbb{N}$ such that $\operatorname{gcd}(k$, ord $(\Gamma))$ is invertible in $R$.

The aim of this section is to prove the multiplicativity of the Adams operation $\psi^{k}$ on $K_{0}(R \# \Gamma)$ (and on the higher $K$-groups) defined in the previous section. Since there is no splitting principle available for $K_{0}(R \# \Gamma)$, this can not be proved as e. g. in [FL]. In place of the splitting principle, we will use a filtration of the exterior power $\Lambda^{i}(M \otimes N)$ of the tensor product of two modules $M$ and $N$ which has been constructed by K. Akin, D. Buchsbaum, and J. Weyman. Each successive quotient in this filtration is isomorphic to a tensor product of (co)Schur modules (see $[\mathrm{ABW}]$ ). Furthermore, we will use a universal form of the Pieri formula established by K. Akin and D. Buchsbaum in $[A B]$ to express (co)Schur modules as polynomials in exterior powers. The results of this section will not be used in the subsequent sections.

Let $\lambda=\left(\lambda_{1} \geq \lambda_{2} \geq \ldots\right) \in \mathbb{N}^{\infty}$ be a partition with $1 \leq|\lambda|:=\lambda_{1}+\lambda_{2}+\cdots<\infty$. The transposed partition (see p. 217 in [ABW]) is denoted by $\tilde{\lambda}=\left(\tilde{\lambda}_{1} \geq \tilde{\lambda}_{2} \geq \ldots\right.$. ). For any f. g. free $R$-module $F$, the Schur module $L_{\lambda}(F)$ is defined as the image of a certain canonical map

$$
d_{\lambda}(F): \Lambda^{\lambda_{1}}(F) \otimes \Lambda^{\lambda_{2}}(F) \otimes \cdots \rightarrow \operatorname{Sym}^{\tilde{\lambda}_{1}}(F) \otimes \operatorname{Sym}^{\tilde{\lambda}_{2}}(F) \otimes \cdots
$$

(see pages 219 and 220 in [ABW]). By Theorem II.2.16 in $[\mathrm{ABW}], L_{\lambda}(F)$ is a $\mathrm{f}$. g. free $R$-module and $L_{\lambda}$ commutes with base change. For any f. g. projective $R$-module $M$, we define the Schur module $L_{\lambda}(M)$ in the same way. By localization, we see that $L_{\lambda}(M)$ is a f. g. projective $R$-module. If furthermore $M$ is an $R \# \Gamma$-module, then the map $d_{\lambda}(M)$ 
obviously is $R \# \Gamma$-linear and $L_{\lambda}(M)$ is an $R \# \Gamma$-module. The same holds for the coSchur module $K_{\lambda}(M)$ defined on p. 220 in [ABW].

Let $s_{\lambda}$ denote the Schur polynomial associated with $\lambda$ (see I $\S 3$ in $[\mathrm{McD}]$ ). It will be viewed as a polynomial in the elementary symmetric functions. It is homogeneous of weight $|\lambda|$. Thus, for any f. g. projective $R \# \Gamma$-module $M$,

$$
s_{\lambda}(M):=s_{\lambda}\left(\left[\Lambda^{1}(M)\right],\left[\Lambda^{2}(M)\right], \ldots\right)
$$

is a well-defined element of $K_{0}\left(\mathcal{M}_{|\lambda|}\right)$ by Lemma 1.2 and Lemma 1.4. Here, the category $\mathcal{M}_{|\lambda|}$ is the category of modules of weight $|\lambda|$ defined in section 1.

Proposition 2.1. Let $M$ be a f. g. projective $R \# \Gamma$-module. Then the Schur module $L_{\lambda}(M)$ and the coSchur module $K_{\lambda}(M)$ are contained in the category $\mathcal{M}_{|\lambda|}$. Furthermore, we have:

$$
\left[L_{\lambda}(M)\right]=s_{\lambda}(M) \quad \text { and } \quad\left[K_{\lambda}(M)\right]=s_{\tilde{\lambda}}(M) \quad \text { in } \quad K_{0}\left(\mathcal{M}_{|\lambda|}\right)
$$

Proof. First, we show that the Schur module $L_{\lambda}(M)$ is contained in $\mathcal{M}_{|\lambda|}$. For this, we use induction on $\lambda$ with respect to lexicographic order (i. e. $\lambda \leq \mu$ iff there is an $r \in \mathbb{N}$ such that $\lambda_{1}=\mu_{1}, \ldots, \lambda_{r}=\mu_{r}$ and $\left.\lambda_{r+1} \leq \mu_{r+1}\right)$. For $\lambda=(1,0,0, \ldots)$, the assertion is trivial. For the induction step, let $\mu$ denote that partition which arises from $\lambda$ by omitting the last column and let $p$ denote the size of the last column in $\lambda\left(\right.$ i. e. $p:=\tilde{\lambda}_{l(\tilde{\lambda})}$ and $\mu_{i}:=\lambda_{i}-1$ for $i=1, \ldots, p$ and $\mu_{i}:=\lambda_{i}$ for $i>p$ ). By the universal form of the Pieri formula (see Theorem (3)(a) in section 3 in $[\mathrm{AB}]$ ), we have for all $\mathrm{f}$. g. free $R$-modules $F$ : There is a filtration on the tensor product $L_{\lambda}(F) \otimes \operatorname{Sym}^{p}(F)$ such that the successive quotients are isomorphic to Schur modules. More precisely, we have: If $\nu$ runs through the set

$$
I:=\left\{\nu \text { partition }: \mu_{i} \leq \nu_{i} \leq \mu_{i}+1 \text { for all } i \text { and }|\nu|=|\lambda|\right\},
$$

then $L_{\nu}(F)$ runs through the sequence of successive quotients. The partition $\lambda$ is obviously the biggest partition in $I$ with respect to lexicographic order; in particular, $L_{\lambda}(F)$ is isomorphic to the smallest (nontrivial) module in the filtration of $L_{\mu}(F) \otimes \operatorname{Sym}^{p}(F)$ (see also section 2 in $[\mathrm{AB}])$. By localization, one easily sees that the corresponding assertions hold for f. g. $R$-projective $R \# \Gamma$-modules as well. By the induction hypothesis and by Lemma 1.2, the module $L_{\mu}(M) \otimes \operatorname{Sym}^{p}(M)$ and the modules $L_{\nu}(M), \nu \in I \backslash\{\lambda\}$, are of weight $|\lambda|$. Thus, all filtration steps and, in particular, $L_{\lambda}(M)$ are of weight $|\lambda|$. Using the corresponding theorem for coSchur modules, one similarly deduces from this that $K_{|\lambda|}(M)$ is of weight $|\lambda|$.

In order to show the equality $\left[L_{\lambda}(M)\right]=s_{\lambda}(M)$, we replace the Pieri formula for $L_{\mu}(M) \otimes$ $\operatorname{Sym}^{p}(M)$ by the Pieri formula for $L_{\mu}(M) \otimes \Lambda^{p}(M)$ (see Theorem (3)(b) in [AB]) and the lexicographic order by the transposed lexicographic order in the procedure above. Then, we immediately obtain a polynomial $s_{\lambda}^{\prime}$ such that $\left[L_{\lambda}(M)\right]=s_{\lambda}^{\prime}\left(\left[\Lambda^{1}(M)\right],\left[\Lambda^{2}(M)\right], \ldots\right)$ in $K_{0}\left(\mathcal{M}_{|\lambda|}\right)$. Since $s_{\lambda}^{\prime}$ does obviously not depend on $R$ and since the equality $s_{\lambda}^{\prime}=s_{\lambda}$ holds for any $\mathbb{Q}$-algebra $R$, we have shown the desired equality $\left[L_{\lambda}(M)\right]=s_{\lambda}(M)$ in $K_{0}\left(\mathcal{M}_{|\lambda|}\right)$. The equality $\left[K_{\lambda}(M)\right]=s_{\tilde{\lambda}}(M)$ can be shown analogously.

For any $i \geq 1$, let $\overline{\mathcal{M}_{i} \otimes \mathcal{M}_{i}}$ be the smallest subcategory of $\mathcal{M}$ which is closed under extensions and kernels of $R \# \Gamma$-epimorphisms and which contains all modules of the form 
$M \otimes N$, where $M, N \in \mathcal{M}_{i}$. The category $\overline{\mathcal{M}_{i} \otimes \mathcal{M}_{i}}$ is obviously contained in $\mathcal{M}_{2 i}$. By Lemma 2.1 in $[\mathrm{Ko} 5], \overline{\mathcal{M}_{1} \otimes \mathcal{M}_{1}}$ is contained in $\mathcal{M}_{1}$. Thus, by Proposition $1.1, \overline{\mathcal{M}_{k} \otimes \mathcal{M}_{k}}$ is contained in $\mathcal{M}_{1}$. In particular, the tensor product defines a ring structur on $K_{0}(R \# \Gamma)$ (in general without 1). Let $P_{i}$ be the universal polynomial defined on page 5 in [FL]. It is homogeneous of weight $i$ in both sets of variables. Thus, for all $\mathrm{f}$. g. projective $R \# \Gamma$-modules $M, N$,

$$
P_{i}(M, N):=P_{i}\left(\left[\Lambda^{1}(M)\right], \ldots,\left[\Lambda^{i}(M)\right] ;\left[\Lambda^{1}(N)\right], \ldots,\left[\Lambda^{i}(N)\right]\right)
$$

is a well-defined element of $K_{0}\left(\overline{\mathcal{M}_{i} \otimes \mathcal{M}_{i}}\right)$ by Lemma 1.2 and Lemma 1.4.

Theorem 2.2. For all f. g. projective $R \# \Gamma$-modules $M, N$ and all $i \geq 1$, the exterior power $\Lambda^{i}(M \otimes N)$ is contained in $\overline{\mathcal{M}_{i} \otimes \mathcal{M}_{i}}$ and we have:

$$
\left[\Lambda^{i}(M \otimes N)\right]=P_{i}(M, N) \quad \text { in } \quad K_{0}\left(\overline{\mathcal{M}_{i} \otimes \mathcal{M}_{i}}\right) .
$$

Proof. By Theorem III.2.4 in $[\mathrm{ABW}]$, there is a filtration on $\Lambda^{i}(M \otimes N)$ by $R \# \Gamma$ submodules with the following property: If $\lambda$ runs through the set of partitions of weight $i$, then the tensor product $L_{\lambda}(M) \otimes K_{\lambda}(M)$ runs through the sequence of successive quotients. (This is proved in $[\mathrm{ABW}]$ only in the non-equivariant situation and only for free $R$-modules $M, N$. The general assertion follows from this using the standard arguments already explained above.) Hence, by Proposition 2.1, all filtration steps and, in particular $\Lambda^{i}(M \otimes N)$, are contained in $\overline{\mathcal{M}_{i} \otimes \mathcal{M}_{i}}$ and we have:

$$
\begin{aligned}
& {\left[\Lambda^{i}(M \otimes N)\right]=\sum_{|\lambda|=i}\left[L_{\lambda}(M) \otimes K_{\lambda}(N)\right]} \\
& \quad=\sum_{|\lambda|=i} s_{\lambda}(M) \cdot s_{\tilde{\lambda}}(N) \\
& \quad=P_{i}(M, N) \quad \text { in } \quad K_{0}\left(\overline{\mathcal{M}_{i} \otimes \mathcal{M}_{i}}\right) .
\end{aligned}
$$

Here, the last equality is formula (4.3') on p. 35 in [McD].

Corollary 2.3. For all $x, y \in K_{0}(R \# \Gamma)$ and all $i \geq 1$, we have

$$
\psi^{i}(x \cdot y)=\psi^{i}(x) \cdot \psi^{i}(y) \quad \text { in } \quad K_{0}\left(\overline{\mathcal{M}_{i} \otimes \mathcal{M}_{i}}\right) .
$$

In particular, $\psi^{k}: K_{0}(R \# \Gamma) \rightarrow K_{0}(R \# \Gamma)$ is a ring homomorphism.

Proof. It obviously suffices to show Corollary 2.3 for elements $x=[M]$ and $y=[N]$ where $M, N$ are f. g. projective $R \# \Gamma$-modules. In this case, Corollary 2.3 follows from Theorem 2.2 and the following identity of polynomials which is easy to prove:

$$
N_{i}\left(P_{j}\left(X_{1}, \ldots, X_{i} ; Y_{1}, \ldots, Y_{i}\right), j=1, \ldots, i\right)=N_{i}\left(X_{1}, \ldots, X_{i}\right) \cdot N_{i}\left(Y_{1}, \ldots, Y_{i}\right) .
$$

The tensor product yields a $K_{0}(R \# \Gamma)$-module structure on Quillen's $K$-groups $K_{q}(R \# \Gamma)$, $q \geq 0$, in the usual way (e. g. see $\S 3$ in $[\mathrm{Q}]$ ).

Corollary 2.4. Let $q \geq 0$. For all $x \in K_{0}(R \# \Gamma)$ and all $y \in K_{q}(R \# \Gamma)$, we have:

$$
\psi^{k}(x \cdot y)=\psi^{k}(x) \cdot \psi^{k}(y) \quad \text { in } \quad K_{q}(R \# \Gamma) .
$$


Proof. For $q=0$, this has been proved in Corollary 2.3. So let $q \geq 1$. For any arbitrary group $G$ let $K_{0}(G, R \# \Gamma)$ denote the Grothendieck group of all representations of the group $G$ on f. g. projective $R \# \Gamma$-modules. As in section 1, one can define a $k$-th Adams operation $\psi^{k}$ on $K_{0}(G, R \# \Gamma)$. Using Quillen's universal transformation, we then obtain a $k$-th Adams operation $\psi^{k}$ on $K_{q}(R \# \Gamma)$ as in section 2 of [Ko4]. As in section 9 of [Gr3], one easily shows that this Adams operation agrees with the Adams operation defined in section 1. Furthermore, one easily proves as in Corollary 2.3 that $\psi^{k}$ on $K_{0}(G, R \# \Gamma)$ is multiplicative. Finally, as in Corollary 2.2a) in [Ko4], one deduces Corollary 2.4 from this using Quillen's universal transformation.

Remark 2.5. If $\Gamma$ acts on $R$ trivially, the tensor product defines a $K_{0}(\Gamma, R)$-module structure on $K_{0}(R \Gamma)$ by Lemma 2.1 in [Ko5]. One easily shows as above that the $k$-th Adams operation $\psi^{k}: K_{0}(R \Gamma) \rightarrow K_{0}(R \Gamma)$ is semilinear with respect to the $k$-th Adams operation on $K_{0}(\Gamma, R)$ defined e. g. in section 1 .

Remark 2.6. Let $l$ be another natural number such that $\operatorname{gcd}(l, \operatorname{ord}(\Gamma))$ is invertible in $R$. One should be able to prove similarly to Corollary 2.3 that $\psi^{k} \circ \psi^{l}=\psi^{k l}$ in $\operatorname{End}\left(K_{0}(R \# \Gamma)\right)$. For this, one should be able to construct a "universal decomposition" of $\Lambda^{k}\left(\Lambda^{l}(M)\right)$ similarly to the universal form of the Cauchy decomposition of $\Lambda^{i}(M \otimes N)$ used in the proof of Theorem 2.2. This problem is called the plethysm problem in the literature and is not yet solved.

If $R$ is a field and $\Gamma$ acts on $R$ trivially, then the Cartan homomorphism $c: K_{0}(R \Gamma) \rightarrow$ $K_{0}(\Gamma, R)$ is injective (see $\S 5$ in $[\mathrm{Ke}]$ ). Thus, the rule $\psi^{k} \circ \psi^{l}=\psi^{k l}$ for $K_{0}(R \Gamma)$ follows from the corresponding rule for $K_{0}(\Gamma, R)$ in this case. If $R$ is the ring of integers in a number field and $\Gamma$ acts on $R$ trivially, the rule $\psi^{k} \circ \psi^{l}=\psi^{k l}$ follows from Theorem 1.3 (1) on p. 99 in $[\mathrm{T}]$ and Theorem 3.7 which is the main result of the next section.

\section{$\S 3$ Power Operations on Locally Free Classgroups}

Let $K$ be number field, $\Gamma$ a finite group, and $k \in \mathbb{N}$ such that $k$ is coprime to the exponent $e(\Gamma)$ of $\Gamma$. Furthermore, we fix a $k^{\prime} \in \mathbb{N}$ with $k k^{\prime} \equiv 1 \bmod e(\Gamma)$. Let $\mathcal{O}_{K}$ denote the ring of integers in $K$ and $\mathrm{Cl}\left(\mathcal{O}_{K} \Gamma\right):=\operatorname{ker}\left(\operatorname{rank}: K_{0}\left(\mathcal{O}_{K} \Gamma\right) \rightarrow \mathbb{Z}\right)$ the locally free classgroup associated with $K$ and $\Gamma$.

In this section, we will prove that the endomorphism $\psi_{k^{\prime}}^{\mathrm{CNT}}$ of $\mathrm{Cl}\left(\mathcal{O}_{K} \Gamma\right)$ defined by $\mathrm{Ph}$. Cassou-Noguès and M. Taylor in [CNT] coincides with the restriction of the symmetric power operation $\sigma^{k}$ on $K_{0}\left(\mathcal{O}_{K} \Gamma\right)$ defined in section 1.

To begin with, we recall some fundamental facts about the locally free classgroup (e. g. see $\S 1$ in Chapter 1 in $[\mathrm{T}])$. Let $K_{0} T\left(\mathcal{O}_{K} \Gamma\right)$ denote the Grothendieck group of all $\mathcal{O}_{K}$-torsion modules over $\mathcal{O}_{K} \Gamma$ which have a $\mathcal{O}_{K} \Gamma$-projective resolution of finite length (and then even of length 1 by Theorem 8 on p. 145 in [Se2]). Let $\partial: K_{1}(K \Gamma) \rightarrow K_{0} T\left(\mathcal{O}_{K} \Gamma\right)$ be the connecting homomorphism; it is uniquely determined by the following property: Let $F$ be a f. g. free $\mathcal{O}_{K} \Gamma$-module and let $\alpha \in \mathrm{GL}(F \otimes K) \cap \operatorname{End}(F)$; then $\partial((F \otimes K, \alpha))=\operatorname{coker}(\alpha$ : $F \rightarrow F)$. Furthermore, let $i_{*}: K_{0} T\left(\mathcal{O}_{K} \Gamma\right) \rightarrow K_{0}\left(\mathcal{O}_{K} \Gamma\right)$ denote the resolution map: it maps a torsion module $M$ with the $\mathcal{O}_{K} \Gamma$-projective resolution $0 \rightarrow P \rightarrow Q \rightarrow M \rightarrow 0$ to 
the element $[P]-[Q]$ in $K_{0}\left(\mathcal{O}_{K} \Gamma\right)$. Then the sequence

$$
K_{1}(K \Gamma) \stackrel{\partial}{\longrightarrow} K_{0} T\left(\mathcal{O}_{K} \Gamma\right) \stackrel{i_{*}}{\longrightarrow} K_{0}\left(\mathcal{O}_{K} \Gamma\right) \stackrel{\text { rank }}{\longrightarrow} \mathbb{Z}
$$

is exact; in particular, we have:

$$
\mathrm{Cl}\left(\mathcal{O}_{K} \Gamma\right) \cong \operatorname{coker}\left(\partial: K_{1}(K \Gamma) \rightarrow K_{0} T\left(\mathcal{O}_{K} \Gamma\right)\right)
$$

Decomposing torsion modules into $\mathfrak{p}$-primary torsion modules induces an isomorphism

$$
K_{0} T\left(\mathcal{O}_{K} \Gamma\right) \cong \underset{\mathfrak{p} \in \operatorname{Max}\left(\mathcal{O}_{K}\right)}{\oplus} K_{0} T\left(\mathcal{O}_{K_{\mathfrak{p}}} \Gamma\right)
$$

here, $K_{\mathfrak{p}}$ denotes the completion of $K$ at $\mathfrak{p}$ and $K_{0} T\left(\mathcal{O}_{K_{\mathfrak{p}}} \Gamma\right)$ is the Grothendieck group of all torsion modules over $\mathcal{O}_{K_{\mathfrak{p}}} \Gamma$ which have a $\mathcal{O}_{K_{\mathfrak{p}}} \Gamma$-free resolution of length 1 . Finally, we have canonical exact (localization) sequences

$$
K_{1}\left(\mathcal{O}_{K_{\mathfrak{p}}} \Gamma\right) \longrightarrow K_{1}\left(K_{\mathfrak{p}} \Gamma\right) \stackrel{\partial}{\longrightarrow} K_{0} T\left(\mathcal{O}_{K_{\mathfrak{p}}} \Gamma\right) \longrightarrow 0, \quad \mathfrak{p} \in \operatorname{Max}\left(\mathcal{O}_{K}\right)
$$

here, $K_{1}\left(\mathcal{O}_{K_{\mathfrak{p}}} \Gamma\right) \rightarrow K_{1}\left(K_{\mathfrak{p}} \Gamma\right)$ is the canonical map and the local connecting homomorphism $\partial: K_{1}\left(K_{\mathfrak{p}} \Gamma\right) \rightarrow K_{0} T\left(\mathcal{O}_{K_{\mathfrak{p}}} \Gamma\right)$ is defined as the global one.

Now we fix a prime ideal $\mathfrak{p} \neq 0$ in $\mathcal{O}_{K}$. Let $\Phi$ denote the composition

$$
\Phi: K_{1}\left(K_{\mathfrak{p}} \Gamma\right) \stackrel{\partial}{\longrightarrow} K_{0} T\left(\mathcal{O}_{K_{\mathfrak{p}}} \Gamma\right) \stackrel{\text { can }}{\longrightarrow} K_{0} T\left(\mathcal{O}_{K} \Gamma\right) \stackrel{i_{*}}{\longrightarrow} K_{0}\left(\mathcal{O}_{K} \Gamma\right) .
$$

Proposition 3.1. Let $\gamma$ be one of the operations $\sigma^{k}, \lambda^{k}, \psi^{k}$ defined in section 1 . Then the following diagram commutes:

$$
\begin{array}{ccc}
K_{1}\left(K_{\mathfrak{p}} \Gamma\right) & \stackrel{\Phi}{\longrightarrow} & K_{0}\left(\mathcal{O}_{K} \Gamma\right) \\
\downarrow \gamma & \downarrow \gamma \\
K_{1}\left(K_{\mathfrak{p}} \Gamma\right) & \stackrel{\Phi}{\longrightarrow} & K_{0}\left(\mathcal{O}_{K} \Gamma\right) .
\end{array}
$$

We will give an algebraic proof and a topological proof of this proposition. The algebraic proof is elementary and explicit, but consists of lengthy computations. The topological proof is conceptual and uses higher $K$-theory. We start with a lemma which will be used in the algebraic proof.

Lemma 3.2. Let $F$ be a f. g. free $\mathcal{O}_{K} \Gamma$-module and $\alpha \in \mathrm{GL}\left(F \otimes K_{\mathfrak{p}}\right)$. We write $\alpha=\beta / \pi$ with $\beta \in \operatorname{End}\left(F \otimes \mathcal{O}_{K_{\mathfrak{p}}}\right) \cap \mathrm{GL}\left(F \otimes K_{\mathfrak{p}}\right)$ and $\pi \in \mathcal{O}_{K} \backslash\{0\}$ and we put

$$
C(\beta):=\operatorname{ker}\left(F \hookrightarrow F \otimes \mathcal{O}_{K_{\mathfrak{p}}} \longrightarrow \operatorname{coker}(\beta)\right) .
$$

Then we have:

(a) $\Phi\left(\left(F \otimes K_{\mathfrak{p}}, \alpha\right)\right)=[C(\beta)]-[F]$ in $K_{0}\left(\mathcal{O}_{K} \Gamma\right)$. 
(b) For all $k_{1}, \ldots, k_{r} \in \mathbb{N}$ with $\operatorname{gcd}\left(k_{1}+\cdots+k_{r}, \operatorname{ord}(\Gamma)\right)=1$ and for all $\beta_{1}, \ldots, \beta_{r} \in$ $\operatorname{End}\left(F \otimes \mathcal{O}_{K_{\mathfrak{p}}}\right) \cap \mathrm{GL}\left(F \otimes K_{\mathfrak{p}}\right)$, the canonical $\mathcal{O}_{K} \Gamma$-linear homomorphism

$$
\operatorname{Sym}^{k_{1}}\left(C\left(\beta_{1}\right)\right) \otimes \cdots \otimes \operatorname{Sym}^{k_{r}}\left(C\left(\beta_{r}\right)\right) \rightarrow C\left(\left(\operatorname{Sym}^{k_{1}}\left(\beta_{1}\right) \otimes \cdots \otimes \operatorname{Sym}^{k_{r}}\left(\beta_{r}\right)\right)\right.
$$

is bijective; here, $\operatorname{Sym}^{k_{1}}\left(\beta_{1}\right) \otimes \cdots \otimes \operatorname{Sym}^{k_{r}}\left(\beta_{r}\right)$ is considered as an endomorphism of $\operatorname{Sym}^{k_{1}}\left(F \otimes \mathcal{O}_{K_{\mathfrak{p}}}\right) \otimes \cdots \otimes \operatorname{Sym}^{k_{r}}\left(F \otimes \mathcal{O}_{K_{\mathfrak{p}}}\right) \cong \operatorname{Sym}^{k_{1}}(F) \otimes \cdots \otimes \operatorname{Sym}^{k_{r}}(F) \otimes \mathcal{O}_{K_{\mathfrak{p}}}$ which is free over $\mathcal{O}_{K_{\mathfrak{p}}} \Gamma$ by Proposition 1.1 .

Proof. (a) In the commutative diagram

$$
\begin{aligned}
& 0 \rightarrow C(\beta) \quad \rightarrow \quad F \quad \rightarrow \operatorname{coker}(\beta) \rightarrow 0 \\
& 0 \rightarrow F \otimes \mathcal{O}_{K_{\mathfrak{p}}} \stackrel{\beta}{\rightarrow} F \otimes \mathcal{O}_{K_{\mathfrak{p}}} \rightarrow \operatorname{coker}(\beta) \rightarrow 0,
\end{aligned}
$$

the composition $F \hookrightarrow F \otimes \mathcal{O}_{K_{\mathfrak{p}}} \longrightarrow \operatorname{coker}(\beta)$ is surjective since $\left(F \otimes \mathcal{O}_{K_{\mathfrak{p}}}\right) / F$ is a divisible abelian group and $\operatorname{coker}(\beta)$ is a f. g. torsion group. Thus, the upper sequence is exact. Since $\operatorname{coker}(\beta)$ is cohomologically trivial, it has $\mathcal{O}_{K} \Gamma$-projective dimension 1 (cf. Proposition 4.1 in $[\mathrm{C}]$ ). Hence, the module $C(\beta)$ is projective over $\mathcal{O}_{K} \Gamma$ and we have

$$
\Phi\left(\left(F \otimes K_{\mathfrak{p}}, \beta\right)\right)=[C(\beta)]-[F] \quad \text { in } \quad K_{0}\left(\mathcal{O}_{K} \Gamma\right) .
$$

For $\beta=\pi$, we obviously have $C(\beta) \cong F$; consequently, $\Phi\left(\left(F \otimes K_{\mathfrak{p}}, \pi\right)\right)=0$. This implies assertion (a).

(b) We write $\operatorname{Sym}^{k}(-)$ for $\operatorname{Sym}^{k_{1}}(-) \otimes \cdots \otimes \operatorname{Sym}^{k_{r}}(-)$. The canonical inclusion $C(\beta) \hookrightarrow$ $F \otimes \mathcal{O}_{K_{\mathfrak{p}}}$ obviously induces an isomorphism $C(\beta) \otimes \mathcal{O}_{K_{\mathfrak{p}}} \stackrel{\sim}{\rightarrow} F \otimes \mathcal{O}_{K_{\mathfrak{p}}}$. Thus, the canonical homomorphism $\operatorname{Sym}^{k}(C(\beta)) \otimes \mathcal{O}_{K_{\mathfrak{p}}} \rightarrow \operatorname{Sym}^{k}\left(F \otimes \mathcal{O}_{K_{\mathfrak{p}}}\right)$ is bijective. Hence, in the commutative diagram with exact rows

$$
\begin{aligned}
& 0 \rightarrow \operatorname{Sym}^{k}(C(\beta)) \quad \stackrel{i}{\rightarrow} \quad \operatorname{Sym}^{k}(F) \quad \rightarrow \quad \operatorname{coker}(i) \quad \rightarrow \quad 0 \\
& \downarrow \quad \| \\
& 0 \rightarrow C\left(\operatorname{Sym}^{k}(\beta)\right) \quad \rightarrow \quad \operatorname{Sym}^{k}(F) \quad \rightarrow \quad \operatorname{coker}\left(\operatorname{Sym}^{k}(\beta)\right) \rightarrow 0 \\
& \downarrow \quad \downarrow \quad \| \\
& 0 \rightarrow \operatorname{Sym}^{k}\left(F \otimes \mathcal{O}_{K_{\mathfrak{p}}}\right) \stackrel{\operatorname{Sym}^{k}(\beta)}{\rightarrow} \operatorname{Sym}^{k}\left(F \otimes \mathcal{O}_{K_{\mathfrak{p}}}\right) \rightarrow \operatorname{coker}\left(\operatorname{Sym}^{k}(\beta)\right) \rightarrow 0,
\end{aligned}
$$

the right vertical composition is bijective. Then, the upper right vertical homomorphism is bijective, too. Hence, the upper left vertical homomorphism is bijective, as was to be shown.

Algebraic Proof of Proposition 3.1. We use additive notation for $K_{1}\left(K_{\mathfrak{p}} \Gamma\right)$. Let $\left(F \otimes K_{\mathfrak{p}}, \beta / \pi\right)$ be an element of $K_{1}\left(K_{\mathfrak{p}} \Gamma\right)$ as in Lemma 3.2. Then the element

$$
\left[\left(F \otimes K_{\mathfrak{p}}, \beta / \pi\right)\right]-\left[\left(F \otimes K_{\mathfrak{p}}, 1\right)\right] \quad \text { of } \quad \tilde{K}_{0}\left(\mathbb{Z}, K_{\mathfrak{p}} \Gamma\right)
$$


is a preimage of $\left(F \otimes K_{\mathfrak{p}}, \beta / \pi\right)$ under the canonical homomorphism $\tilde{K}_{0}\left(\mathbb{Z}, K_{\mathfrak{p}} \Gamma\right) \rightarrow$ $K_{1}\left(K_{\mathfrak{p}} \Gamma\right)$ (see also Theorem 1.6(f)). Using Theorem 1.6(c)(i) and (f), Lemma 3.2(a), Lemma 3.2(b), Theorem 1.6(c)(i), and Lemma 3.2(a) successively, we obtain:

$$
\begin{aligned}
\Phi & \left.\sigma^{k}\left(\left(F \otimes K_{\mathfrak{p}}, \beta / \pi\right)\right)\right)= \\
& =\Phi\left(\sum _ { \substack { a , b _ { 1 } , \ldots , b _ { u } \geq 1 \\
a + b _ { 1 } + \cdots + b _ { u } = k } } ( - 1 ) ^ { u } \left(\operatorname{Sym}^{a}\left(F \otimes K_{\mathfrak{p}}\right) \otimes \operatorname{Sym}^{b_{1}}\left(F \otimes K_{\mathfrak{p}}\right) \otimes \cdots \otimes \operatorname{Sym}^{b_{u}}\left(F \otimes K_{\mathfrak{p}}\right),\right.\right. \\
& =\sum_{\substack{a, b_{1}, \ldots, b_{u} \geq 1 \\
a+b_{1}+\cdots+b_{u}=k}}(-1)^{u}\left(\left[C\left(\operatorname{Sym}^{a}(\beta) \otimes 1 \otimes \cdots \otimes 1\right)\right]-\right. \\
& {\left[\sum _ { \substack { a , b _ { 1 } , \ldots , b _ { u } \geq 1 \\
a + b _ { 1 } + \cdots + b _ { u } = k } } ( - 1 ) ^ { u } \left(\left[\operatorname{Sym}^{a}(C(\beta)) \otimes \operatorname{Sym}^{b_{1}}(F) \otimes \cdots \otimes \operatorname{Sym}^{b_{u}}(F)\right]-\right.\right.} \\
& \left.\left.\left.=\sum_{\substack{a \geq 0, b_{1}, \ldots, b_{u} \geq 1 \\
a+b_{1}+\cdots+b_{u}=k}}^{a}(F) \otimes \operatorname{Sym}^{b_{1}}(F) \otimes \cdots \otimes \operatorname{Sym}^{b_{u}}(F)\right]\right)\right] \\
= & \sigma^{k}([C(\beta)]-[F]) \\
= & \sigma^{k}\left(\Phi\left(\left(F \otimes \operatorname{Sym}^{a}(F) \otimes \operatorname{Sym}^{b_{1}}(F) \otimes \cdots \otimes \operatorname{Sym}^{b_{u}}(F)\right]\right)\right. \\
& \left.\left.\left(K_{\mathfrak{p}}, \beta / \pi\right)\right)\right) .
\end{aligned}
$$

This proves Proposition 3.1 for $\gamma=\sigma^{k}$. For $\gamma=\lambda^{k}$ or $\gamma=\psi^{k}$, Proposition 3.1 can be shown analogously or can be deduced from the assertion for $\gamma=\sigma^{k}$ using Theorem 1.6(c) and (d).

Topological Proof of Proposition 3.1. Let $K_{0} T_{\mathrm{pr}}\left(\mathcal{O}_{K_{\mathrm{p}}} \Gamma\right)$ denote the Grothendieck group of all torsion modules over $\mathcal{O}_{K_{\mathfrak{p}}} \Gamma$ which have a $\mathcal{O}_{K_{\mathrm{p}}} \Gamma$-projective resolution of length 1. Then the canonical homomorphism $K_{0} T\left(\mathcal{O}_{K_{\mathrm{p}}} \Gamma\right) \rightarrow K_{0} T_{\mathrm{pr}}\left(\mathcal{O}_{K_{\mathrm{p}}} \Gamma\right)$ is bijective. The injectivity has been proved in Theorem 1(ii) on p. 3 in [F2]. The surjectivity follows from the fact that, in the commutative diagram

$$
\begin{array}{ccc}
K_{1}\left(K_{\mathfrak{p}} \Gamma\right) & \stackrel{\partial}{\rightarrow} & K_{0} T\left(\mathcal{O}_{K_{\mathfrak{p}}} \Gamma\right) \\
\| & \downarrow & \\
K_{1}\left(K_{\mathfrak{p}} \Gamma\right) & \stackrel{\partial}{\rightarrow} & K_{0} T_{\mathrm{pr}}\left(\mathcal{O}_{K_{\mathfrak{p}}} \Gamma\right) \stackrel{i_{*}}{\rightarrow} K_{0}\left(\mathcal{O}_{K_{\mathfrak{p}}} \Gamma\right) \stackrel{\text { can }}{\rightarrow} K_{0}\left(K_{\mathfrak{p}} \Gamma\right),
\end{array}
$$

the lower sequence is exact (cf. Theorem 1(ii) on p. 3 in [F2]) and that the canonical homomorphism $K_{0}\left(\mathcal{O}_{K_{\mathfrak{p}}} \Gamma\right) \rightarrow K_{0}\left(K_{\mathfrak{p}} \Gamma\right)$ is injective (cf. Theorem 6.1 in [Sw]). In the section "The localization theorem for projective modules" in [Gr1], it is shown that $K_{0} T_{\mathrm{pr}}\left(\mathcal{O}_{K_{\mathrm{p}}} \Gamma\right)$ is isomorphic to the group of connected components of the homotopy fibre of the canoncial continuous map from the $K$-theory space associated with $\mathcal{O}_{K_{\mathfrak{p}}} \Gamma$ to the 
$K$-theory space associated with $K_{\mathfrak{p}} \Gamma$. Thus, the continuous map $\sigma^{k}$ on the $K$-theory space associated with $\mathcal{O}_{K_{\mathfrak{p}}} \Gamma$ defined in section 1 induces a symmetric power operation $\sigma^{k}$ on $K_{0} T_{\mathrm{pr}}\left(\mathcal{O}_{K_{\mathrm{p}}} \Gamma\right)$ which, in particular, commutes with the connecting homomorphism $\partial: K_{1}\left(K_{\mathfrak{p}} \Gamma\right) \rightarrow K_{0} T_{\mathrm{pr}}\left(\mathcal{O}_{K_{\mathfrak{p}}} \Gamma\right)$. Anologously, the Grothendieck group $K_{0} T_{\mathfrak{p}}\left(\mathcal{O}_{K} \Gamma\right)$ of all $\mathfrak{p}-$ torsion modules over $\mathcal{O}_{K} \Gamma$ of $\mathcal{O}_{K} \Gamma$-projective dimension 1 carries a symmetric power operation $\sigma^{k}$ which commutes with the localization isomorphism $K_{0} T_{\mathfrak{p}}\left(\mathcal{O}_{K} \Gamma\right) \stackrel{\sim}{\rightarrow} K_{0} T_{\mathrm{pr}}\left(\mathcal{O}_{K_{\mathfrak{p}}} \Gamma\right)$ and with the resolution map $i_{*}: K_{0} T_{\mathfrak{p}}\left(\mathcal{O}_{K} \Gamma\right) \rightarrow K_{0}\left(\mathcal{O}_{K} \Gamma\right)$. Since $\Phi$ coincides with the composition

$$
K_{1}\left(K_{\mathfrak{p}} \Gamma\right) \stackrel{\partial}{\rightarrow} K_{0} T_{\mathrm{pr}}\left(\mathcal{O}_{K_{\mathfrak{p}}} \Gamma\right) \stackrel{\sim}{\leftarrow} K_{0} T_{\mathfrak{p}}\left(\mathcal{O}_{K} \Gamma\right) \stackrel{i_{*}}{\rightarrow} K_{0}\left(\mathcal{O}_{K} \Gamma\right)
$$

(cf. section 8 in [Gr2]), Proposition 3.1 is proved for $\sigma^{k}$. The assertions for $\lambda^{k}$ and $\psi^{k}$ can be deduced from this as in the algebraic proof.

Remark 3.3. Let $X$ be a scheme and $U$ an open subscheme of $X$. As in the proof above, one can define power operations on the homotopy fibre of the canonical continuous map from the $K$-theory space of $X$ to the $K$-theory space of $U$. In the section "The localization theorem ..." in [Gr1], it is shown that this homotopy fibre is homotopy equivalent to the $K$-theory space associated with the category $\mathcal{H}$ consisting of all quasi-coherent sheaves on $X$ which are zero on $U$ and which have a resolution by vector bundles on $X$ of length 1. It is likely that one can use the ideas developed in the algebraic proof to describe these operations on the $K$-theory space of $\mathcal{H}$ simplicially, and, finally, on $K_{0}(\mathcal{H})$ algebraically, i. e. in terms of modules. I hope to say more on this in a future paper.

Let $\mathcal{M}_{1}, \mathcal{M}_{2}, \ldots$ be the categories defined in section 1 .

Proposition 3.4. For any $1 \leq i \leq k-1$, the composition

$$
\mathrm{Cl}\left(\mathcal{O}_{K} \Gamma\right) \times \mathrm{Cl}\left(\mathcal{O}_{K} \Gamma\right) \stackrel{\sigma^{i} \times \sigma^{k-i}}{\longrightarrow} K_{0}\left(\mathcal{M}_{i}\right) \times K_{0}\left(\mathcal{M}_{k-i}\right) \stackrel{\otimes}{\longrightarrow} K_{0}\left(\mathcal{O}_{K} \Gamma\right)
$$

is the zero map. In particular, the restriction of $\sigma^{k}: K_{0}\left(\mathcal{O}_{K} \Gamma\right) \rightarrow K_{0}\left(\mathcal{O}_{K} \Gamma\right)$ to the locally free classgroup $\mathrm{Cl}\left(\mathcal{O}_{K} \Gamma\right)$ is an homomorphism. Analogous assertions hold for exterior power operations, too.

Proof. Let $(x, y) \in \mathrm{Cl}\left(\mathcal{O}_{K} \Gamma\right) \times \mathrm{Cl}\left(\mathcal{O}_{K} \Gamma\right)$. Let $P_{1}, P_{2}, Q_{1}, Q_{2}$ be f. g. projective $\mathcal{O}_{K} \Gamma$ modules such that $x=\left[P_{1}\right]-\left[Q_{1}\right]$ and $y=\left[P_{2}\right]-\left[Q_{2}\right]$. By Theorem $\mathrm{A}$ in $[\mathrm{Sw}]$, we may assume that $P_{1}, P_{2}, Q_{1}, Q_{2}$ are projective left ideals in $\mathcal{O}_{K} \Gamma$. Then it suffices by Theorem 1.6(c)(i) to show Proposition 3.4 for elements $x, y$ of the form $x=\left[\mathcal{O}_{K} \Gamma\right]-[I]$ and $y=\left[\mathcal{O}_{K} \Gamma\right]-[J]$ where $I, J$ are projective left ideals in $\mathcal{O}_{K} \Gamma$. Furthermore, we may assume by Theorem A in $[\mathrm{Sw}]$ that the annihilators of the torsion moduls $\mathcal{O}_{K} \Gamma / I$ and $\mathcal{O}_{K} \Gamma / J$ are relatively prime. By Theorem 1.6(c)(i), we have in $K_{0}\left(\mathcal{M}_{i}\right)$ :

$$
\begin{aligned}
\sigma^{i}(x) & =\sum_{\substack{a \geq 0, b_{1}, \ldots, b_{u} \geq 1 \\
a+b_{1}+\cdots+b_{u}=i}}(-1)^{u}\left[\operatorname{Sym}^{a}\left(\mathcal{O}_{K} \Gamma\right) \otimes \operatorname{Sym}^{b_{1}}(I) \otimes \cdots \otimes \operatorname{Sym}^{b_{u}}(I)\right]= \\
& =\sum_{\substack{a, b_{1}, \ldots, b_{u} \geq 1 \\
a+b_{1}+\cdots+b_{u}=i}}(-1)^{u}\left(\left[\operatorname{Sym}^{a}\left(\mathcal{O}_{K} \Gamma\right) \otimes \operatorname{Sym}^{b_{1}}(I) \otimes \cdots \otimes \operatorname{Sym}^{b_{u}}(I)\right]-\right.
\end{aligned}
$$

$$
\left.\left[\operatorname{Sym}^{a}(I) \otimes \operatorname{Sym}^{b_{1}}(I) \otimes \cdots \otimes \operatorname{Sym}^{b_{u}}(I)\right]\right)
$$


For any $a \geq 1$ and for any prime ideal $\mathfrak{p} \neq 0$ in $\mathcal{O}_{K}$ which does not lie in the support of $\mathcal{O}_{K} \Gamma / I$, the localization of the canonical homomorphism

$$
\operatorname{Sym}^{a}(I) \rightarrow \operatorname{Sym}^{a}\left(\mathcal{O}_{K} \Gamma\right)
$$

at $\mathfrak{p}$ is bijective. In particular, $\operatorname{Sym}^{a}(I) \rightarrow \operatorname{Sym}^{a}\left(\mathcal{O}_{K} \Gamma\right)$ is injective and the support of the torsion module $\operatorname{Sym}^{a}\left(\mathcal{O}_{K} \Gamma\right) / \operatorname{Sym}^{a}(I)$ is contained in the support of the torsion module $\mathcal{O}_{K} \Gamma / I$. The same holds for $y$ and $J$. Consequently, it suffices to show the following assertion: Let $M_{1} \subseteq N_{1}$ and $M_{2} \subseteq N_{2}$ be modules in $\mathcal{M}_{i}$ and $\mathcal{M}_{k-i}$, respectively, such that $N_{1} / M_{1}$ and $N_{2} / M_{2}$ are torsion modules whose annihilators are relatively prime. Then we have:

$$
\left(\left[N_{1}\right]-\left[M_{1}\right]\right) \cdot\left(\left[N_{2}\right]-\left[M_{2}\right]\right)=0 \quad \text { in } \quad K_{0}\left(\mathcal{O}_{K} \Gamma\right) .
$$

This immediately follows from the fact that, in the commutative diagram with exact rows

$$
\begin{aligned}
& 0 \rightarrow M_{1} \otimes M_{2} \rightarrow N_{1} \otimes M_{2} \rightarrow N_{1} / M_{1} \otimes M_{2} \rightarrow 0 \\
& 0 \rightarrow M_{1} \otimes N_{2} \quad \rightarrow \quad N_{1} \otimes N_{2} \quad \rightarrow \quad N_{1} / M_{1} \otimes N_{2} \rightarrow 0
\end{aligned}
$$

the right vertical homomorphism is bijective.

The equality $\sigma^{k}(x+y)=\sum_{i=0}^{k} \sigma^{i}(x) \cdot \sigma^{k-i}(y)$ (for $x, y \in \operatorname{Cl}\left(\mathcal{O}_{K} \Gamma\right)$ ) now shows that $\sigma^{k}: \mathrm{Cl}\left(\mathcal{O}_{K} \Gamma\right) \rightarrow \mathrm{Cl}\left(\mathcal{O}_{K} \Gamma\right)$ is an homomorphism.

As for Proposition 3.1, we give a topological proof for Proposition 3.4 with a more conceptual argument.

Topological proof of Proposition 3.4 (Sketch). We will only show that the multiplication

$$
\mathrm{Cl}\left(\mathcal{O}_{K} \Gamma\right) \times \mathrm{Cl}\left(\mathcal{O}_{K} \Gamma\right) \rightarrow K_{0}\left(\mathcal{O}_{K} \Gamma\right)
$$

is trivial. The general statement can then be proved by blowing up the following argument as in the algebraic proof.

Similarly as in the topological proof of Proposition 3.1, the tensor product induces a multiplication on the various $K$-theory spaces and, in particular, on the $K$-groups $K_{1}\left(K_{\mathfrak{p}} \Gamma\right)$, $K_{0} T\left(\mathcal{O}_{K} \Gamma\right), K_{0}\left(\mathcal{O}_{K} \Gamma\right), \ldots$ which are compatible with the maps in the local or global localization sequences. On $K_{1}\left(K_{\mathfrak{p}} \Gamma\right)$ (as on all higher $K$-groups), this multiplication is linear. Hence, it is trivial. Since the connecting homomorphism $\partial: K_{1}\left(K_{\mathfrak{p}} \Gamma\right) \rightarrow$ $K_{0} T\left(\mathcal{O}_{K_{\mathfrak{p}}} \Gamma\right)$ is surjective, the multiplication on $K_{0} T\left(\mathcal{O}_{K_{\mathfrak{p}}} \Gamma\right)$ is trivial, too. As in the algebraic proof, one shows furthermore that for all prime ideals $\mathfrak{p} \neq \mathfrak{q}$ in $\mathcal{O}_{K}$, the multiplication $K_{0} T_{\mathfrak{p}}\left(\mathcal{O}_{K} \Gamma\right) \times K_{0} T_{\mathfrak{q}}\left(\mathcal{O}_{K} \Gamma\right) \rightarrow K_{0}\left(\mathcal{O}_{K} \Gamma\right)$ is trivial. Altogether, the multiplication $K_{0} T\left(\mathcal{O}_{K} \Gamma\right) \times K_{0} T\left(\mathcal{O}_{K} \Gamma\right) \rightarrow K_{0}\left(\mathcal{O}_{K} \Gamma\right)$ is trivial. This implies that the multiplication on $\mathrm{Cl}\left(\mathcal{O}_{K} \Gamma\right)$ is trivial, as was to be shown.

Now let $\gamma$ be one of the operations $\sigma^{k}, \lambda^{k}$, or $\psi^{k}$ defined in section 1. For any prime ideal $\mathfrak{p} \neq 0$ in $\mathcal{O}_{K}$, the operation $\gamma$ commutes with the canonical homomorphism $K_{1}\left(\mathcal{O}_{K_{\mathfrak{p}}} \Gamma\right) \rightarrow$ $K_{1}\left(K_{\mathfrak{p}} \Gamma\right)$ by Theorem 1.6(b) and hence induces an operation $\gamma$ on $K_{0} T\left(\mathcal{O}_{K_{\mathfrak{p}}} \Gamma\right)$. Let $\gamma$ also denote the direct sum of these operations on $K_{0} T\left(\mathcal{O}_{K} \Gamma\right) \cong \oplus_{\mathfrak{p}} K_{0} T\left(\mathcal{O}_{K_{\mathfrak{p}}} \Gamma\right)$. Then, $\gamma$ 
commutes with the connecting homomorphism $\partial: K_{1}(K \Gamma) \rightarrow K_{0} T\left(\mathcal{O}_{K} \Gamma\right)$ by Theorem 1.6(b). Consequently, $\gamma$ induces an operation on $\mathrm{Cl}\left(\mathcal{O}_{K} \Gamma\right) \cong \operatorname{coker}(\partial)$ denoted by $\gamma$ again. On the other hand, the operation $\gamma$ on $K_{0}\left(\mathcal{O}_{K} \Gamma\right)$ obviously induces an operation $\gamma$ on $\mathrm{Cl}\left(\mathcal{O}_{K} \Gamma\right)$ by restricting.

Corollary 3.5. These two definitions of the operation $\gamma$ on $\mathrm{Cl}\left(\mathcal{O}_{K} \Gamma\right)$ conincide.

Proof. This immediately follows from Propositions 3.1 and 3.4.

For any prime ideal $\mathfrak{p} \neq 0$ in $\mathcal{O}_{K}$, let $G_{\mathfrak{p}}:=\operatorname{Gal}\left(\bar{K}_{\mathfrak{p}} / K_{\mathfrak{p}}\right)$ denote the absolute Galois group. By Chapter 1 in $[\mathrm{T}]$, the pairing

$$
\begin{aligned}
K_{0}\left(\bar{K}_{\mathfrak{p}} \Gamma\right) \times K_{1}\left(\bar{K}_{\mathfrak{p}} \Gamma\right) & \rightarrow \bar{K}_{\mathfrak{p}}^{\times} \\
([P],(M, \alpha)) & \mapsto \operatorname{det}_{\bar{K}_{\mathfrak{p}}}\left(\left.\operatorname{Hom}_{\bar{K}_{\mathfrak{p}} \Gamma}(P, \alpha)\right|_{\operatorname{Hom}_{\bar{K}_{\mathfrak{p}} \Gamma}(P, M)}\right)
\end{aligned}
$$

induces an isomorphism

$$
K_{1}\left(K_{\mathfrak{p}} \Gamma\right) \stackrel{\sim}{\rightarrow} \operatorname{Hom}_{G_{\mathfrak{p}}}\left(K_{0}\left(\bar{K}_{\mathfrak{p}} \Gamma\right), \bar{K}_{\mathfrak{p}}^{\times}\right) .
$$

Note that the determinant map used here coincides with the determinant map used in [T] (see Proposition 2.3 in [Que]). We identify the Grothendieck group $K_{0}\left(\bar{K}_{\mathfrak{p}} \Gamma\right)$ with the classical ring of virtual characters of $\Gamma$. Let $\langle-,-\rangle: K_{0}\left(\bar{K}_{\mathfrak{p}} \Gamma\right) \times K_{0}\left(\bar{K}_{\mathfrak{p}} \Gamma\right) \rightarrow \mathbb{Z}$ denote the classical character pairing.

Now let $k$ be an arbitrary natural number. Let $\hat{\psi}^{k} \in \operatorname{End}_{G_{\mathfrak{p}}}\left(K_{0}\left(\bar{K}_{\mathfrak{p}} \Gamma\right)\right)$ denote the adjoint of the Adams operation $\psi^{k}$ with respect to $\langle-,-\rangle$.

Lemma 3.6. (a) For all characters $\chi \in K_{0}\left(\bar{K}_{\mathfrak{p}} \Gamma\right)$ and all $\gamma \in \Gamma$, we have:

$$
\hat{\psi}^{k}(\chi)(\gamma)=\sum_{\tau^{k}=\gamma} \chi(\tau) \quad \text { in } \quad \bar{K}_{\mathfrak{p}}
$$

In particular, we have $\hat{\psi}^{k}=\psi^{k^{\prime}}$ if $\operatorname{gcd}(k, \operatorname{ord}(\Gamma))=1$ and $k k^{\prime} \equiv 1 \bmod e(\Gamma)$.

(b) Let $\Gamma$ be an abelian group. Let $\phi: \bar{K}_{\mathfrak{p}} \Gamma \rightarrow \bar{K}_{\mathfrak{p}} \Gamma$ denote the ring homomorphism given by $\gamma \mapsto \gamma^{k}$. Then we have for all f. g. $\bar{K}_{\mathfrak{p}} \Gamma$-modules $M$ :

$$
\hat{\psi}^{k}([M])=\left[\left(\bar{K}_{\mathfrak{p}} \Gamma\right)_{\phi} \otimes_{\bar{K}_{\mathfrak{p}} \Gamma} M\right] \quad \text { in } \quad K_{0}\left(\bar{K}_{\mathfrak{p}} \Gamma\right) .
$$

Proof. (a) Let the class function $\tilde{\psi}^{k}(\chi): \Gamma \rightarrow \mathbb{C}$ be given by $\tilde{\psi}^{k}(\chi)(\gamma)=\sum_{\tau^{k}=\gamma} \chi(\tau)$ for $\gamma \in \Gamma$. Then we have for all characters $\theta \in K_{0}(\mathbb{C} \Gamma)$ :

$$
\begin{aligned}
& \left\langle\tilde{\psi}^{k}(\chi), \theta\right\rangle=\frac{1}{\operatorname{ord}(\Gamma)} \sum_{\gamma \in \Gamma} \tilde{\psi}^{k}(\chi)(\gamma) \overline{\theta(\gamma)} \\
& =\frac{1}{\operatorname{ord}(\Gamma)} \sum_{\gamma \in \Gamma} \sum_{\tau^{k}=\gamma} \chi(\tau) \overline{\theta(\gamma)}=\frac{1}{\operatorname{ord}(\Gamma)} \sum_{\tau \in \Gamma} \chi(\tau) \overline{\theta\left(\tau^{k}\right)} \\
& =\frac{1}{\operatorname{ord}(\Gamma)} \sum_{\tau \in \Gamma} \chi(\tau) \overline{\psi^{k}(\theta)(\tau)}=\left\langle\chi, \psi^{k}(\theta)\right\rangle=\left\langle\hat{\psi}^{k}(\chi), \theta\right\rangle .
\end{aligned}
$$

(Here, we have used Proposition (12.8) on p. 317 in [CR].) This shows the main assertion of Lemma 3.6(a). If $\operatorname{gcd}(k, \operatorname{ord}(\Gamma))=1$, then we have $\left\{\tau \in \Gamma: \tau^{k}=\gamma\right\}=\left\{\gamma^{k^{\prime}}\right\}$ for all 
$\gamma \in \Gamma$. This proves $\hat{\psi}^{k}=\psi^{k^{\prime}}$ in this case.

(b) For all $\bar{K}_{\mathfrak{p}} \Gamma$-modules $N$, we have:

$$
\begin{array}{r}
\left\langle\left(\bar{K}_{\mathfrak{p}} \Gamma\right)_{\phi} \otimes_{\bar{K}_{\mathfrak{p}} \Gamma} M, N\right\rangle=\operatorname{dim}_{\bar{K}_{\mathfrak{p}}} \operatorname{Hom}_{\bar{K}_{\mathfrak{p}} \Gamma}\left(\left(\bar{K}_{\mathfrak{p}} \Gamma\right)_{\phi} \otimes_{\bar{K}_{\mathfrak{p}} \Gamma} M, N\right)= \\
\quad=\operatorname{dim}_{\bar{K}_{\mathfrak{p}}} \operatorname{Hom}_{\bar{K}_{\mathfrak{p}} \Gamma}\left(M, \psi^{k}(N)\right)=\left\langle M, \psi^{k}(N)\right\rangle=\left\langle\hat{\psi}^{k}(M), N\right\rangle .
\end{array}
$$

This proves Lemma 3.6(b).

We now assume that the prime divisors of ord $(\Gamma)$ are unramified in $K$. Furthermore, let $k$ be odd if $\Gamma$ has irreducible symplectic characters. Under these assumptions, Ph. CassouNoguès and M. Taylor have shown that the endomorphisms $\psi_{k}^{\mathrm{CNT}}:=\operatorname{Hom}\left(\psi^{k}, \bar{K}_{\mathfrak{p}}^{\times}\right)$of $K_{1}\left(\bar{K}_{\mathfrak{p}} \Gamma\right) \cong \operatorname{Hom}_{G_{\mathfrak{p}}}\left(K_{0}\left(\bar{K}_{\mathfrak{p}} \Gamma\right), \bar{K}_{\mathfrak{p}}^{\times}\right), \mathfrak{p} \in \operatorname{Max}\left(\mathcal{O}_{K}\right)$, induce an endomorphism on $\mathrm{Cl}\left(\mathcal{O}_{K} \Gamma\right)$ denoted by $\psi_{k}^{\mathrm{CNT}}$ again (cf. Chapter 9 in $[\mathrm{T}]$ ). In the paper [BC], D. Burns and T. Chinburg have raised the question whether there is an algebraic interpretation of this endomorphism. The following theorem gives an affirmative answer to this question in the case $\operatorname{gcd}(k, \operatorname{ord}(\Gamma))=1$. For this, let $\sigma^{k}$ and $\psi^{k}$ denote (the restriction of) the symmetric power operation and $k$-th Adams operation on $\mathrm{Cl}\left(\mathcal{O}_{K} \Gamma\right) \subset K_{0}\left(\mathcal{O}_{K} \Gamma\right)$ constructed in section 1 .

Theorem 3.7. Let $\operatorname{gcd}(k, \operatorname{ord}(\Gamma))=1$ and $k^{\prime} \in \mathbb{N}$ such that $k k^{\prime} \equiv 1 \bmod e(\Gamma)$. Then we have:

$$
\psi_{k^{\prime}}^{\mathrm{CNT}}=\sigma^{k} \quad \text { on } \quad \mathrm{Cl}\left(\mathcal{O}_{K} \Gamma\right)
$$

and

$$
k \cdot \psi_{k^{\prime}}^{\mathrm{CNT}} \oplus \mathrm{id}=\psi^{k} \quad \text { on } \quad \operatorname{Cl}\left(\mathcal{O}_{K} \Gamma\right) \oplus \mathbb{Z}\left[\mathcal{O}_{K} \Gamma\right]=K_{0}\left(\mathcal{O}_{K} \Gamma\right) .
$$

Proof. By Theorem 3.3 in [Ko4], the operation $\lambda^{k}$ on $K_{1}\left(\bar{K}_{\mathfrak{p}} \Gamma\right)$ corresponds to the endomorphism $(-1)^{k-1} \operatorname{Hom}\left(\hat{\psi}^{k}, \bar{K}_{\mathfrak{p}}^{\times}\right)$of $\operatorname{Hom}_{G_{\mathfrak{p}}}\left(K_{0}\left(\bar{K}_{\mathfrak{p}} \Gamma\right), \bar{K}_{\mathfrak{p}}^{\times}\right)$via the isomorphism

$$
K_{1}\left(\bar{K}_{\mathfrak{p}} \Gamma\right) \cong \operatorname{Hom}_{G_{\mathfrak{p}}}\left(K_{0}\left(\bar{K}_{\mathfrak{p}} \Gamma\right), \bar{K}_{\mathfrak{p}}^{\times}\right) .
$$

Thus, the operation $\sigma^{k}$ corresponds to the endomorphism $\operatorname{Hom}\left(\psi^{k^{\prime}}, \bar{K}_{\mathfrak{p}}^{\times}\right)$by Theorem 1.6(d)(ii) and Lemma 3.6(a). By construction (see Chapter 9 in $[\mathrm{T}]), \psi_{k^{\prime}}^{\mathrm{CNT}}$ on $\mathrm{Cl}\left(\mathcal{O}_{K} \Gamma\right)$ is induced by $\operatorname{Hom}\left(\psi^{k^{\prime}}, \bar{K}_{\mathfrak{p}}^{\times}\right)$on $K_{1}\left(\bar{K}_{\mathfrak{p}} \Gamma\right), \mathfrak{p} \in \operatorname{Max}\left(\mathcal{O}_{K}\right)$. Now the equality $\psi_{k^{\prime}}^{\text {CNT }}=\sigma^{k}$ on $\mathrm{Cl}\left(\mathcal{O}_{K} \Gamma\right)$ follows from Corollary 3.5. The equality $k \cdot \psi_{k^{\prime}}^{\mathrm{CNT}}=\psi^{k}$ on $\mathrm{Cl}\left(\mathcal{O}_{K} \Gamma\right)$ can be shown analogously. Finally, the equality $\psi^{k}=$ id on $\mathbb{Z}\left[\mathcal{O}_{K} \Gamma\right] \subseteq K_{0}\left(\mathcal{O}_{K} \Gamma\right)$ has already been shown in Theorem 1.6(e).

Corollary 3.8. We have $\sigma^{k}=\sigma^{k+e(\Gamma)}$ on $\operatorname{Cl}\left(\mathcal{O}_{K} \Gamma\right)$.

Proof. This follows from Theorem 1.3(2) on p. 99 in [T] and Theorem 3.7.

The following corollary answers the question (1.12) in Chapter 9 of [T].

Corollary 3.9. Let $c: \mathrm{Cl}\left(\mathcal{O}_{K} \Gamma\right) \hookrightarrow K_{0}\left(\mathcal{O}_{K} \Gamma\right) \rightarrow K_{0}\left(\Gamma, \mathcal{O}_{K}\right)$ denote the Cartan homomorphism. Then the following diagrams commute:

$$
\begin{array}{rrrrrr}
\mathrm{Cl}\left(\mathcal{O}_{K} \Gamma\right) & \stackrel{c}{\rightarrow} & K_{0}\left(\Gamma, \mathcal{O}_{K}\right) & \mathrm{Cl}\left(\mathcal{O}_{K} \Gamma\right) \oplus \mathbb{Z}\left[\mathcal{O}_{K} \Gamma\right] & \stackrel{c}{\rightarrow} & K_{0}\left(\Gamma, \mathcal{O}_{K}\right) \\
\downarrow \psi_{k^{\prime}}^{\mathrm{CNT}} & & \downarrow \sigma^{k} & \downarrow k \cdot \psi_{k^{\prime}}^{\mathrm{CNT}} \oplus \mathrm{id} & \\
\mathrm{Cl}\left(\mathcal{O}_{K} \Gamma\right) & \stackrel{c}{\rightarrow} & K_{0}\left(\Gamma, \mathcal{O}_{K}\right) & \mathrm{Cl}\left(\mathcal{O}_{K} \Gamma\right) \oplus \mathbb{Z}\left[\mathcal{O}_{K} \Gamma\right] & \stackrel{c}{\rightarrow} & K_{0}\left(\Gamma, \mathcal{O}_{K}\right) .
\end{array}
$$


Proof. This immediately follows from Theorem 1.6(a) and Theorem 3.7. In addition to the algebraic or topolgical arguments used in the proof of Theorem 3.7, we give a geometric proof of this corollary since Proposition 3.10 which will be used in this proof is of independent interest.

By Theorem 1.3(3) on p. 99 in $[\mathrm{T}]$, the following diagram commutes for any prime ideal $\mathfrak{p} \neq 0$ in $\mathcal{O}_{K}:$

$$
\begin{array}{rlrl}
K_{0} T\left(\mathcal{O}_{K_{\mathfrak{p}}} \Gamma\right) \stackrel{c}{\longrightarrow} K_{0} T\left(\Gamma, \mathcal{O}_{K_{\mathfrak{p}}}\right) \cong K_{0}\left(\Gamma, \mathcal{O}_{K} / \mathfrak{p}\right) \\
\downarrow \psi_{k^{\prime}}^{\mathrm{CNT}} & & \downarrow \psi^{k} \\
K_{0} T\left(\mathcal{O}_{K_{\mathfrak{p}}} \Gamma\right) \stackrel{c}{\longrightarrow} K_{0} T\left(\Gamma, \mathcal{O}_{K_{\mathfrak{p}}}\right) \cong K_{0}\left(\Gamma, \mathcal{O}_{K} / \mathfrak{p}\right) .
\end{array}
$$

Now Corollary 3.9 follows from the subsequent Proposition 3.10 (and Theorem 1.6(e)) where, for the first diagram in Corollary 3.9, we in addition use the fact that, for all prime ideals $\mathfrak{p} \neq \mathfrak{q}$ in $\mathcal{O}_{K}$ and all $x \in K_{0}\left(\Gamma, \mathcal{O}_{K} / \mathfrak{p}\right), y \in K_{0}\left(\Gamma, \mathcal{O}_{K} / \mathfrak{q}\right)$, the product $i_{*}(x) \cdot i_{*}(y)$ vanishes in $K_{0}\left(\Gamma, \mathcal{O}_{K}\right)$ (cf. the end of the (algebraic) proof of Proposition 3.4).

Proposition 3.10. For any prime ideal $\mathfrak{p} \neq 0$ in $\mathcal{O}_{K}$ and any $j \geq 0$, the following diagrams commute:

$$
\begin{aligned}
& K_{0}\left(\Gamma, \mathcal{O}_{K} / \mathfrak{p}\right) \stackrel{i_{*}}{\rightarrow} K_{0}\left(\Gamma, \mathcal{O}_{K}\right) \quad K_{0}\left(\Gamma, \mathcal{O}_{K} / \mathfrak{p}\right) \stackrel{i_{*}}{\rightarrow} K_{0}\left(\Gamma, \mathcal{O}_{K}\right) \\
& \downarrow \psi^{j} \quad \downarrow \sigma^{j} \quad \downarrow j \cdot \psi^{j} \quad \downarrow \psi^{j} \\
& K_{0}\left(\Gamma, \mathcal{O}_{K} / \mathfrak{p}\right) \stackrel{i_{*}}{\rightarrow} K_{0}\left(\Gamma, \mathcal{O}_{K}\right) \quad K_{0}\left(\Gamma, \mathcal{O}_{K} / \mathfrak{p}\right) \stackrel{i_{*}}{\rightarrow} K_{0}\left(\Gamma, \mathcal{O}_{K}\right) .
\end{aligned}
$$

Here, $i_{*}$ denotes the composition of the canonical map $K_{0}\left(\Gamma, \mathcal{O}_{K} / \mathfrak{p}\right) \rightarrow K_{0}^{\prime}\left(\mathcal{O}_{K} \Gamma\right)$ with the isomorphism $K_{0}^{\prime}\left(\mathcal{O}_{K} \Gamma\right) \cong K_{0}\left(\Gamma, \mathcal{O}_{K}\right)$ (cf. Notations).

Proof. The conormal module $\mathfrak{p} / \mathfrak{p}^{2}$ of the closed immersion $i: \operatorname{Spec}\left(\mathcal{O}_{K} / \mathfrak{p}\right) \hookrightarrow \operatorname{Spec}\left(\mathcal{O}_{K}\right)$ is trivial. Thus, the Bott multiplier $\theta^{j}(i):=1+\left[\mathfrak{p} / \mathfrak{p}^{2}\right]+\cdots+\left[\left(\mathfrak{p} / \mathfrak{p}^{2}\right)^{j-1}\right]$ associated with $i$ equals $j$ in $K_{0}\left(\Gamma, \mathcal{O}_{K} / \mathfrak{p}\right)$. Now, the commutativity of the second diagram follows from the equivariant Adams-Riemann-Roch theorem without denominators (see Corollary (5.2) in [Ko1]). The commutativity of the first diagram follows from the (more general) equivariant Riemann-Roch theorem (see Satz (5.1) in [Ko1]) applied to the natural operation $\sigma^{j}$. For this, we have to show

$$
\sigma^{j}\left(\mathfrak{p} / \mathfrak{p}^{2}, x\right)=\psi^{j}(x) \quad \text { in } \quad K_{0}\left(\Gamma, \mathcal{O}_{K} / \mathfrak{p}\right)
$$

for all $x \in K_{0}\left(\Gamma, \mathcal{O}_{K} / \mathfrak{p}\right)$. Here, $\sigma^{j}\left(\mathfrak{p} / \mathfrak{p}^{2}, x\right)$ is defined as follows (see section 5 in [Ko1]): Let the polynomial ring $K_{0}\left(\Gamma, \mathcal{O}_{K} / \mathfrak{p}\right)[N]$ be equipped with the unique (special) $\lambda$-ring structure such that $K_{0}\left(\Gamma, \mathcal{O}_{K} / \mathfrak{p}\right)$ is a $\lambda$-subring and such that $N$ is of $\lambda$-degree 1 ; then, $\sigma^{j}\left(\mathfrak{p} / \mathfrak{p}^{2}, x\right)$ is defined to be the value of the polynomial $\sigma^{j}(x \cdot(1-N)) /(1-N)$ at the place $N=1$. As for exterior powers (see p. 5 in [FL]), there is a universal polynomial $Q_{j} \in \mathbb{Z}\left[X_{1}, \ldots, X_{j} ; Y_{1}, \ldots, Y_{j}\right]$ such that

$$
\sigma^{j}(x \cdot(1-N))=Q_{j}\left(\sigma^{1}(x), \ldots, \sigma^{j}(x) ; \sigma^{1}(1-N), \ldots, \sigma^{j}(1-N)\right) .
$$


Since $\sigma^{i}(1-N)=1-N$ for all $i \geq 0$ and since $Q_{j}$ is homogeneous of weight $j$ in both sets of variables, we have:

$$
\sigma^{j}\left(\mathfrak{p} / \mathfrak{p}^{2}, x\right)=Q_{j}\left(\sigma^{1}(x), \ldots, \sigma^{j}(x) ; 0, \ldots, 0,1\right) \quad \text { in } \quad K_{0}\left(\Gamma, \mathcal{O}_{K} / \mathfrak{p}\right) .
$$

If $L_{1}, \ldots, L_{n}$ are of modules of $\operatorname{rank} 1, M:=L_{1} \oplus \cdots \oplus L_{n}$, and $N$ is an arbitrary module, we have:

$$
\begin{aligned}
Q_{j} & \left(\left[\operatorname{Sym}^{1}(M)\right], \ldots,\left[\operatorname{Sym}^{j}(M)\right] ;\left[\operatorname{Sym}^{1}(N)\right], \ldots,\left[\operatorname{Sym}^{j}(N)\right]\right)= \\
& =\left[\operatorname{Sym}^{j}\left(\left(L_{1} \oplus \cdots \oplus L_{n}\right) \otimes N\right)\right] \\
& =\left[\operatorname{Sym}^{j}\left(L_{1} \otimes N \oplus \cdots \oplus L_{n} \otimes N\right)\right] \\
& =\sum_{b_{1}+\cdots+b_{n}=j}\left[\operatorname{Sym}^{b_{1}}\left(L_{1} \otimes N\right) \otimes \cdots \otimes \operatorname{Sym}^{b_{n}}\left(L_{n} \otimes N\right)\right] \\
& =\sum_{b_{1}+\cdots+b_{n}=j}\left[L_{1}^{\otimes b_{1}} \otimes \cdots \otimes L_{n}^{\otimes b_{n}} \otimes \operatorname{Sym}^{b_{1}}(N) \otimes \cdots \otimes \operatorname{Sym}^{b_{n}}(N)\right] .
\end{aligned}
$$

Thus, for $x=\sum_{i=1}^{n} l_{i}$ with elements $l_{i}$ of $\lambda$-degree 1 , we have:

$$
Q_{j}\left(\sigma^{1}(x), \ldots, \sigma^{j}(x) ; Y_{1}, \ldots, Y_{j}\right)=\sum_{b_{1}+\cdots+b_{n}=j} l_{1}^{b_{1}} \cdots l_{n}^{b_{n}} \cdot Y_{b_{1}} \cdots Y_{b_{n}}
$$

(with $Y_{i}:=0$ for $i>j$ ) and, hence,

$$
Q_{j}\left(\sigma^{1}(x), \ldots, \sigma^{j}(x) ; 0, \ldots, 0,1\right)=l_{1}^{j}+\cdots+l_{n}^{j}=\psi^{j}(x) .
$$

Using the splitting principle (see section (2.5) in [Ko1]), we finally obtain the equality $\sigma^{j}\left(\mathfrak{p} / \mathfrak{p}^{2}, x\right)=\psi^{j}(x)$ for an arbitrary $x \in K_{0}\left(\Gamma, \mathcal{O}_{K} / \mathfrak{p}\right)$. This completes the proof of Proposition 3.10.

Remark 3.11. The construction of $\psi_{k}^{\mathrm{CNT}}$ in [T] only shows that, for any prime ideal $\mathfrak{p} \neq 0$ in $\mathcal{O}_{K}$, the image of the canoncial homomorphism

$$
K_{1}\left(\mathcal{O}_{K_{\mathfrak{p}}} \Gamma\right) \rightarrow K_{1}\left(K_{\mathfrak{p}} \Gamma\right) \cong \operatorname{Hom}_{G_{\mathfrak{p}}}\left(K_{0}\left(\bar{K}_{\mathfrak{p}} \Gamma\right), \bar{K}_{\mathfrak{p}}^{\times}\right)
$$

is invariant under the endomorphism $\operatorname{Hom}\left(\psi^{k}, \bar{K}_{\mathfrak{p}}^{\times}\right)$. If $p:=\operatorname{char}\left(\mathcal{O}_{K} / \mathfrak{p}\right)$ does not divide $\operatorname{gcd}(k, \operatorname{ord}(\Gamma))$, the considerations above even show that there exists an endomorphism of $K_{1}\left(\mathcal{O}_{K_{\mathfrak{p}}} \Gamma\right)$ which is compatible with $\operatorname{Hom}\left(\psi^{k}, \bar{K}_{\mathfrak{p}}^{\times}\right)$on $K_{1}\left(K_{\mathfrak{p}} \Gamma\right)$ and that the assumption " $K_{\mathfrak{p}}$ is absolutely unramified" used in [T] is not necessary for this. There remains open the question whether, in the case $p \mid \operatorname{gcd}(k, \operatorname{ord}(\Gamma))$, there exists an endomorphism of $K_{1}\left(\mathcal{O}_{K_{\mathfrak{p}}} \Gamma\right)$ which is compatible with $\operatorname{Hom}\left(\psi^{k}, \bar{K}_{\mathfrak{p}}^{\times}\right)$. This question has an affirmative answer in the following two (extreme) cases:

(a) Let $\Gamma$ be an abelian group. Let $\phi: \mathcal{O}_{K_{\mathfrak{p}}} \Gamma \rightarrow \mathcal{O}_{K_{\mathfrak{p}}} \Gamma$ denote the ring homomorphism given by $\gamma \mapsto \gamma^{k}$. Then $\phi$ induces an endomorphism on $K_{1}\left(\mathcal{O}_{K_{\mathfrak{p}}} \Gamma\right)$ which is compatible with $\operatorname{Hom}\left(\psi^{k}, \bar{K}_{\mathfrak{p}}^{\times}\right)$on $K_{1}\left(K_{\mathfrak{p}} \Gamma\right)$. This is easy to prove or follows from formula (3.18) on p. 113 in [T] which holds for arbitrary abelian groups and not only for $p$-groups.

(b) Let $k$ be equal to the exponent of $\Gamma$. Let $\epsilon: \mathcal{O}_{K_{\mathfrak{p}}} \Gamma \rightarrow \mathcal{O}_{K_{\mathfrak{p}}} \Gamma, \sum a_{\gamma}[\gamma] \mapsto \sum a_{\gamma}[1]$, denote the augmentation homomorphism. Then $\epsilon$ induces an endomorphism on $K_{1}\left(\mathcal{O}_{K_{\mathfrak{p}}} \Gamma\right)$ which is compatible with $\operatorname{Hom}\left(\psi^{k}, \bar{K}_{\mathfrak{p}}^{\times}\right)$on $K_{1}\left(K_{\mathfrak{p}} \Gamma\right)$, as one can easily show. 
Remark 3.12. In the definition of $\psi_{k}^{\mathrm{CNT}}$, it has been assumed that $k$ is odd if $\Gamma$ has irreducible symplectic characters. This assumption is obviously implied by the assumption $\operatorname{gcd}(k, \operatorname{ord}(\Gamma))=1$. Thus, the description of $\psi_{k}^{\mathrm{CNT}}$ given in this section does not yield any improvement into this direction. The following considerations about $K_{1}(\mathbb{R} \Gamma)$ will make clear why this assumption is necessary.

Let $j$ denote complex conjugation and let $K_{0}(\mathbb{C} \Gamma)=K_{0}^{\mathbb{R}} \oplus K_{0}^{\mathbb{C}} \oplus K_{0}^{\mathbb{H}}$ be the decomposition of the classical ring $K_{0}(\mathbb{C} \Gamma)$ of virtual characters introduced in I, section 12.6 in [Se1]. Then the subgroup $K_{0}^{\mathrm{s}}$ of $K_{0}(\mathbb{C} \Gamma)$ generated by symplectic characters has the following description:

$$
K_{0}^{\mathrm{s}}=2 K_{0}^{\mathbb{R}} \oplus(1+j) K_{0}^{\mathbb{C}} \oplus K_{0}^{\mathbb{H}}=(1+j) K_{0}(\mathbb{C} \Gamma)+K_{0}^{\mathbb{H}} .
$$

Furthermore, we have a canonical isomorphism

$$
K_{1}(\mathbb{R} \Gamma) \cong \operatorname{Hom}_{j}^{+}\left(K_{0}(\mathbb{C} \Gamma), \mathbb{C}^{\times}\right)
$$

where $\operatorname{Hom}_{j}^{+}\left(K_{0}(\mathbb{C} \Gamma), \mathbb{C}^{\times}\right)$denotes the group of all Galois-invariant homomorphisms $f$ : $K_{0}(\mathbb{C} \Gamma) \rightarrow \mathbb{C}^{\times}$with $f\left(K_{0}^{\mathrm{s}}\right) \subseteq \mathbb{R}_{+}^{\times}$(see Chapter 1 in $[\mathrm{T}]$ ). Now, the assertion that a Galois-invariant operation $\gamma$ on $K_{0}(\mathbb{C} \Gamma)$ induces an operation on $K_{1}(\mathbb{R} \Gamma)$ is equivalent to the assertion that $\gamma$ maps the subgroup $K_{0}^{\mathbb{H}}$ into $K_{0}^{\mathrm{s}}$. If $\Gamma$ is the quaternion group $Q_{8}$ and $\gamma=\psi^{2}$, the latter assertion is not true as one can easily show; i. e. the analogue of $\psi_{2}^{\mathrm{CNT}}$ can not be defined on $K_{1}\left(\mathbb{R} Q_{8}\right)$ (and $K_{1}\left(K Q_{8}\right)$ ). However, we have

$$
\hat{\psi}^{k}\left(K_{0}^{\mathbb{H}}\right) \subseteq K_{0}^{\mathrm{s}}
$$

for all $k \geq 1$. This follows from the fact that $\operatorname{Hom}\left(\hat{\psi}^{k}, \mathbb{C}^{\times}\right)$corresponds to the operation $\sigma^{k}$ on $K_{1}(\mathbb{R} \Gamma)$ (see the proof of Theorem 3.7). Alternatively, this can be proved in the following elementary way: Let $S \in K_{0}^{\mathbb{H}}$ be an irreducible $\mathbb{C} \Gamma$-module. Then we have to show that, for all irreducible $V \in K_{0}^{\mathbb{R}}$, the number $\left\langle V, \hat{\psi}^{k}(S)\right\rangle$ is even and that, for all irreducible $V \in K_{0}^{\mathbb{C}}$, the equality $\left\langle V, \hat{\psi}^{k}(S)\right\rangle=\left\langle j(V), \hat{\psi}^{k}(S)\right\rangle$ holds. The first statement follows from the fact that, for all f. g. $\mathbb{R} \Gamma$-modules $W$, the dimension $\langle\mathbb{C} \otimes W, S\rangle=$ $\operatorname{dim}_{\mathbb{C}} \operatorname{Hom}_{\mathbb{C} \Gamma}(\mathbb{C} \otimes W, S)$ is even since $\operatorname{Hom}_{\mathbb{R} \Gamma}(W, S)$ is a vector space over $\mathbb{H}$. The second statement is clear since $j$ fixes $S$.

Remark 3.13. Let $\Gamma$ be an abelian group, $\mathfrak{p} \in \operatorname{Max}\left(\mathcal{O}_{K}\right)$, and $\phi_{k}: K_{\mathfrak{p}} \Gamma \rightarrow K_{\mathfrak{p}} \Gamma$ given by $\gamma \mapsto \gamma^{k}$. Pulling back the module structure along $\phi_{k}$ defines an endomorphism $\phi_{k}^{*}$ on $K_{1}\left(\Gamma, K_{\mathfrak{p}}\right)=K_{1}\left(K_{\mathfrak{p}} \Gamma\right)$. Then we have

$$
\phi_{k}^{*}=\sigma^{k} \quad \text { on } \quad K_{1}\left(K_{\mathfrak{p}} \Gamma\right)
$$

for all $k \geq 1$. This follows from Lemma 3.6(b) and the fact that the operation $\sigma^{k}$ corresponds to $\operatorname{Hom}\left(\hat{\psi}^{k}, \bar{K}_{\mathfrak{p}}^{\times}\right)$via the isomorphism $K_{1}\left(K_{\mathfrak{p}} \Gamma\right) \cong \operatorname{Hom}_{G_{\mathfrak{p}}}\left(K_{0}\left(\bar{K}_{\mathfrak{p}} \Gamma\right), \bar{K}_{\mathfrak{p}}^{\times}\right)$. Note that $\phi_{k}^{*}$ can not be defined on $K_{1}\left(\mathcal{O}_{K_{\mathrm{p}}} \Gamma\right)$ in general since a projective $\mathcal{O}_{K_{\mathrm{p}}} \Gamma$-module need not to be projective again after pulling back the module structure along $\phi_{k}$. Thus, it is unlikely that the endomorphism $\sigma^{p}=\operatorname{Hom}\left(\hat{\psi}^{p}, K_{\mathfrak{p}}^{\times}\right)$of $K_{1}\left(K_{\mathfrak{p}} \Gamma\right)$ induces an endomorphism of $K_{0} T\left(\mathcal{O}_{K_{\mathfrak{p}}} \Gamma\right)\left(p:=\operatorname{char}\left(\mathcal{O}_{K} / \mathfrak{p}\right)\right)$. 


\section{$\S 4$ The Galois Structure of the Module of Relative Differentials}

Let $N / K$ be a Galois extension of number fields with Galois group $\Gamma=\operatorname{Gal}(N / K)$. Let $\Omega:=\Omega_{\mathcal{O}_{N} / \mathcal{O}_{K}}^{1}$ denote the module of relative differentials, $\mathfrak{D}:=\operatorname{Ann}_{\mathcal{O}_{N}}(\Omega)$ the different, $V:=\operatorname{supp}(\Omega)$ the set of ramified primes in $\operatorname{Spec}\left(\mathcal{O}_{N}\right)$, and $\mathfrak{J}$ the $\Gamma$-stable ideal $\prod_{\mathfrak{P} \in V} \mathfrak{P}$ in $\mathcal{O}_{N}$.

The aim of this section is to prove the following theorem which will be used in section 5 to compute the tangential element $T_{f}$ associated with the morphism $f: \operatorname{Spec}\left(\mathcal{O}_{N}\right) \rightarrow$ $\operatorname{Spec}\left(\mathcal{O}_{K}\right)$.

Theorem 4.1. We have:

$$
[\Omega]=[\mathfrak{J}]-[\mathfrak{J} \mathfrak{D}] \quad \text { in } \quad K_{0}^{\prime}\left(\mathcal{O}_{N} \# \Gamma\right)
$$

Proof. The quotient module $\mathfrak{J} / \mathfrak{J} \mathfrak{D}$ decomposes into the direct sum of the $\Gamma$-modules $\mathfrak{J}_{\mathfrak{p}} / \mathfrak{J}_{\mathfrak{p}} \mathfrak{D}_{\mathfrak{p}}, \mathfrak{p} \in f(V)$, over $\mathcal{O}_{N}$. Thus, we have:

$$
[\mathfrak{J}]-[\mathfrak{J} \mathfrak{D}]=\sum_{\mathfrak{p} \in f(V)}\left[\mathfrak{J}_{\mathfrak{p}} / \mathfrak{J}_{\mathfrak{p}} \mathfrak{D}_{\mathfrak{p}}\right] \quad \text { in } \quad K_{0}^{\prime}\left(\mathcal{O}_{N} \# \Gamma\right)
$$

Likewise, we have:

$$
[\Omega]=\sum_{\mathfrak{p} \in f(V)}\left[\Omega_{\mathfrak{p}}\right] \quad \text { in } \quad K_{0}^{\prime}\left(\mathcal{O}_{N} \# \Gamma\right) .
$$

Therefore, it suffices to show that, for all $\mathfrak{p} \in f(V)$, we have

$$
\left[\Omega_{\mathfrak{p}}\right]=\left[\mathfrak{J}_{\mathfrak{p}} / \mathfrak{J}_{\mathfrak{p}} \mathfrak{D}_{\mathfrak{p}}\right]
$$

in the Grothendieck group $K_{0} T\left(\Gamma, \mathcal{O}_{N, \mathfrak{p}}\right)$ of all f. g. torsion modules over $\mathcal{O}_{N, \mathfrak{p}} \# \Gamma$.

Now we fix a $\mathfrak{p} \in f(V)$ and a $\mathfrak{P} \in V$ over $\mathfrak{p}$. Let $\Gamma_{\mathfrak{P}} \subseteq \Gamma$ denote the decomposition group of $\mathfrak{P}$. Since the canonical homomorphism $K_{0} T\left(\mathcal{O}_{N, \mathfrak{p}} \# \Gamma\right) \rightarrow K_{0} T\left(\mathcal{O}_{N, \mathfrak{P}} \# \Gamma_{\mathfrak{P}}\right)$ is bijective, it suffices to show the equality

$$
\left[\Omega_{\mathfrak{P}}\right]=\left[\mathfrak{P O} \mathcal{O}_{N, \mathfrak{P}} / \mathfrak{P} \mathfrak{D}_{\mathfrak{P}}\right] \quad \text { in } \quad K_{0} T\left(\Gamma_{\mathfrak{P}}, \mathcal{O}_{N, \mathfrak{P}}\right)
$$

We now write $\Gamma, \mathcal{O}_{N}, \mathfrak{P}, \Omega$, and $\mathfrak{D}$ for $\Gamma_{\mathfrak{P}}, \mathcal{O}_{N, \mathfrak{P}}, \mathfrak{P} \mathcal{O}_{N, \mathfrak{P}}, \Omega_{\mathfrak{P}}$, and $\mathfrak{D}_{\mathfrak{P}}$, respectively. Let $\pi \in \mathfrak{P}$ be a prime element and let $L \subseteq N$ denote the inertia field associated with $\mathfrak{P}$. We again write $\mathcal{O}_{L}$ for the localization of $\mathcal{O}_{L}$ in $\mathfrak{P} \cap L$. By Proposition 18 on p. 19 in [Se2], the $\mathcal{O}_{L}$-algebra $\mathcal{O}_{N}$ is generated by $\pi$. Thus, the module $\Omega_{\mathcal{O}_{N} / \mathcal{O}_{L}}$ of relative differentials is generated by $d \pi$. Since $\mathcal{O}_{L}$ is unramified over $\mathcal{O}_{K, \mathfrak{p}}$, the canonical homomorphism $\Omega_{\mathcal{O}_{N} / \mathcal{O}_{L}} \rightarrow \Omega$ is bijective. Hence, $\Omega$ is generated by $d \pi$, too. Let the unit $u_{\gamma} \in \mathcal{O}_{N}^{\times}$be defined by $\gamma(\pi)=u_{\gamma} \cdot \pi$ for each $\gamma \in \Gamma$. Then, for all $i \geq 1$, we have $\gamma\left(\pi^{i}\right)=u_{\gamma}^{i} \cdot \pi^{i}$ and, hence,

$$
\gamma\left(\pi^{i} d \pi\right)=u_{\gamma}^{i+1} \pi^{i} d \pi+u_{\gamma}^{i} \pi^{i+1} d u_{\gamma} \quad \text { in } \quad \Omega .
$$

Consequently, we have:

$$
\gamma\left(\overline{\pi^{i} d \pi}\right)=u_{\gamma}^{i+1} \overline{\pi^{i} d \pi} \quad \text { in } \quad \mathfrak{P}^{i} \Omega / \mathfrak{P}^{i+1} \Omega .
$$


Thus, the sequence

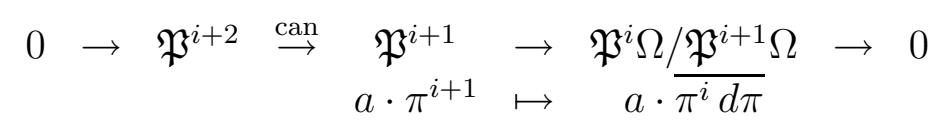

$\left(a \in \mathcal{O}_{N}\right)$ is an exact sequence of $\Gamma$-modules over $\mathcal{O}_{N}$ for all $i=0, \ldots, l-1$ (here, $l$ denotes the length of $\Omega$, i. e. $\mathfrak{D}=\mathfrak{P}^{l}$ ). This implies the desired equality

$$
[\Omega]=\sum_{i=0}^{l-1}\left[\mathfrak{P}^{i} \Omega / \mathfrak{P}^{i+1} \Omega\right]=\sum_{i=1}^{l}\left[\mathfrak{P}^{i} / \mathfrak{P}^{i+1}\right]=[\mathfrak{P} / \mathfrak{P D}] \quad \text { in } \quad K_{0} T\left(\Gamma, \mathcal{O}_{N}\right) .
$$

Corollary 4.2. If $N / K$ is a tame extension, we have

$$
[\Omega]=\left[\mathfrak{D}^{-1}\right]-\left[\mathcal{O}_{N}\right] \text { in } K_{0}^{\prime}\left(\mathcal{O}_{N} \# \Gamma\right) .
$$

Proof. By Theorem (2.6) on p. 210 in [N], we have

$$
\mathfrak{J} \mathfrak{D}=\prod_{\mathfrak{P} \in V} \mathfrak{P}^{e_{\mathfrak{P}}}
$$

where $e_{\mathfrak{P}}$ denotes the ramification index at $\mathfrak{P}$. Thus, for all $\mathfrak{p} \in f(V)$, the $\Gamma$-stable ideal $\mathfrak{J}_{\mathfrak{p}} \mathfrak{D}_{\mathfrak{p}}$ is equal to $\mathfrak{p} \mathcal{O}_{N, \mathfrak{p}}$ and then isomorphic to $\mathcal{O}_{N, \mathfrak{p}}$ as a $\Gamma$-module over $\mathcal{O}_{N, \mathfrak{p}}$. Thus, we have:

$$
[\Omega]=\sum_{\mathfrak{p} \in f(V)}\left[\Omega_{\mathfrak{p}}\right]=\sum_{\mathfrak{p} \in f(V)}\left[\Omega_{\mathfrak{p}} \otimes(\mathfrak{J} \mathfrak{D})_{\mathfrak{p}}^{-1}\right]=\left[\Omega \otimes(\mathfrak{J} \mathfrak{D})^{-1}\right] \quad \text { in } \quad K_{0} T\left(\Gamma, \mathcal{O}_{N}\right) .
$$

By Theorem 4.1, we finally have:

$$
[\Omega]=\left[\Omega \otimes(\mathfrak{J} \mathfrak{D})^{-1}\right]=([\mathfrak{J}]-[\mathfrak{J} \mathfrak{D}]) \cdot\left([\mathfrak{J D}]^{-1}\right)=\left[\mathfrak{D}^{-1}\right]-\left[\mathcal{O}_{N}\right] \quad \text { in } \quad K_{0}^{\prime}\left(\mathcal{O}_{N} \# \Gamma\right) .
$$

The subsequent Remark 4.3 and Example 4.4 contain situations where not only the equality $[\Omega]=\left[\mathfrak{D}^{-1}\right]-\left[\mathcal{O}_{N}\right]$ holds in the Grothendieck group $K_{0}^{\prime}\left(\mathcal{O}_{N} \# \Gamma\right)$, but $\Omega$ and $\mathfrak{D}^{-1} / \mathcal{O}_{N}$ are even isomorphic equivariantly.

Remark 4.3. Let $\mathfrak{P} \in \operatorname{Spec}\left(\mathcal{O}_{N}\right)$ be a tamely ramified prime ideal and let $I \subseteq \Gamma$ denote the inertia group associated with $\mathfrak{P}$. Then the modules $\Omega_{\mathfrak{P}}$ and $\mathfrak{D}_{\mathfrak{P}}^{-1} / \mathcal{O}_{N, \mathfrak{P}}$ are isomorphic as $I$-modules over $\mathcal{O}_{N, \mathfrak{P}}$.

Proof. Let $L:=N^{I}$ denote the inertia field associated with $\mathfrak{P}, \mathfrak{p}:=\mathfrak{P} \cap L$ the prime below $\mathfrak{P}$, and $e$ the ramification index at $\mathfrak{P}$. Furthermore, let $\hat{\mathcal{O}}_{N, \mathfrak{P}}$ and $\hat{\mathcal{O}}_{L, \mathfrak{p}}$ denote the respective completions. Then there is a prime element $\pi \in \hat{\mathcal{O}}_{N, \mathfrak{P}}$ such that $\pi^{e} \in \hat{\mathcal{O}}_{L, \mathfrak{p}}$ (e. g. see p. 26 in [F1]). As in the proof of Theorem 4.1, the module of relative differentials $\Omega_{\mathfrak{P}} \cong \Omega_{\mathcal{O}_{N, \mathfrak{P}} / \mathcal{O}_{L, \mathfrak{p}}} \cong \Omega_{\hat{\mathcal{O}}_{N, \mathfrak{P}} / \hat{\mathcal{O}}_{L, \mathfrak{p}}}$ is generated by $d \pi$. Let the $e$-th root of unity $\zeta_{\gamma}$ defined by $\gamma(\pi)=\zeta_{\gamma} \cdot \pi$ for each $\gamma \in I$. Since $\zeta_{\gamma}$ is contained in $\hat{\mathcal{O}}_{L, \mathfrak{p}}$ (e. g. see p. 26 in [F1]), we have:

$$
\gamma(d \pi)=\zeta_{\gamma} d \pi+\pi d \zeta_{\gamma}=\zeta_{\gamma} d \pi \quad \text { in } \quad \Omega_{\mathfrak{P}}
$$


Hence, the sequence

$$
\begin{aligned}
& 0 \rightarrow \mathfrak{P}^{e} \hat{\mathcal{O}}_{N, \mathfrak{P}} \rightarrow \mathfrak{P} \hat{\mathcal{O}}_{N, \mathfrak{P}} \rightarrow \Omega_{\mathfrak{P}} \rightarrow 0 \\
& a \cdot \pi \mapsto a \cdot d \pi
\end{aligned}
$$

$\left(a \in \mathcal{O}_{N}\right)$ is an exact sequence of $I$-modules over $\hat{\mathcal{O}}_{N, \mathfrak{P}}$. The homomorphism $\mathfrak{D}_{\mathfrak{P}}^{-1} \rightarrow$ $\mathfrak{P} \hat{\mathcal{O}}_{N, \mathfrak{P}}, a \mapsto a \cdot \pi^{e}$, of $I$-modules over $\mathcal{O}_{N, \mathfrak{P}}$ now induces the desired isomorphism

$$
\mathfrak{D}_{\mathfrak{P}}^{-1} / \mathcal{O}_{N, \mathfrak{P}} \stackrel{\sim}{\rightarrow} \Omega_{\mathfrak{P}}
$$

of $I$-modules over $\mathcal{O}_{N, \mathfrak{P}}$.

Example 4.4. Let $D$ be a square-free natural number, $K$ the field of rational numbers, and $N:=\mathbb{Q}(\sqrt{D})$. Then the modules $\Omega$ and $\mathfrak{D}^{-1} / \mathcal{O}_{N}$ are isomorphic as $\Gamma$-modules over $\mathcal{O}_{N}$.

Proof. We have

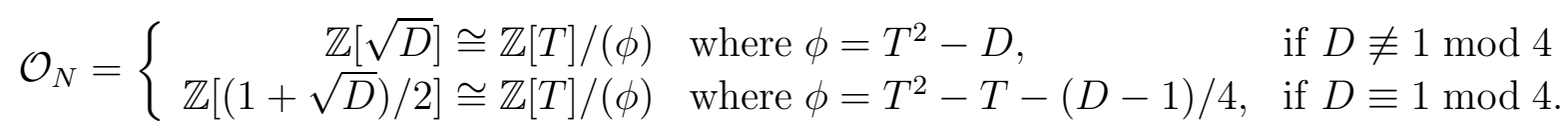

Let $t:=\sqrt{D}$ or $t:=(1+\sqrt{D}) / 2$. By Satz (2.4) on p. 207 in $[\mathrm{N}]$, the different $\mathfrak{D}_{N / K}$ is generated by $\phi^{\prime}(t)$ which is equal to $2 \sqrt{D}$ or $\sqrt{D}$. Let $\gamma \in \Gamma=\operatorname{Gal}(N / K)$ denote the non-trivial automorphism. Then we have $\gamma(t)=-t$ or $\gamma(t)=-t+1$ and hence

$$
\gamma(d t)=-d t \quad \text { in } \quad \Omega
$$

in both cases. Consequently, the sequence

$$
0 \rightarrow \mathcal{O}_{N} \stackrel{\text { can }}{\rightarrow} \begin{array}{cl}
\mathfrak{D}^{-1} & \rightarrow \Omega \\
a \cdot\left(\phi^{\prime}(t)^{-1}\right) & \mapsto a \cdot d t
\end{array} \rightarrow 0
$$

$\left(a \in \mathcal{O}_{N}\right)$ is an exact sequence of $\Gamma$-modules over $\mathcal{O}_{N}$. This proves Example 4.4.

\section{$\S 5$ Equivariant Adams-Riemann-Roch Formulas for Tame Galois Extensions}

Let $N / K$ be a tame Galois extension of number fields with Galois group $\Gamma=\mathrm{Gal}(N / K)$. Let $f: \operatorname{Spec}\left(\mathcal{O}_{N}\right) \rightarrow \operatorname{Spec}\left(\mathcal{O}_{K}\right)$ denote the $\Gamma$-morphism associated with $N / K$. As in section 3 , let $\psi_{k}^{\mathrm{CNT}}$ denote the endomorphism of the locally free classgroup $\mathrm{Cl}\left(\mathcal{O}_{K} \Gamma\right)$ defined by $\mathrm{Ph}$. Cassou-Noguès and M. Taylor in [CNT].

Any $\Gamma$-stable ideal $\mathfrak{A}$ in $N$ defines an element $(\mathfrak{A}) \in \mathrm{Cl}\left(\mathcal{O}_{K}\right)$ in the obvious way (see below). In the paper $[\mathrm{BC}]$, D. Burns and T. Chinburg have established a formula for $\psi_{k}^{\mathrm{CNT}}((\mathfrak{A}))$. In this section, we will give a geometric interpretation of this formula: On the one hand, we will apply the equivariant Adams-Riemann-Roch formula of [Ko3] to the 
$\Gamma$-morphism $f$ using Corollary 4.2. On the other hand, we will reformulate the formula of Burns and Chinburg using Theorem 3.7. It will then turn out, that, more or less, the formula of Burns and Chinburg, is a strengthening of the equivariant Adams-RiemannRoch formula in this situation.

Since $\mathcal{O}_{N}$ and $\mathcal{O}_{K}$ are regular rings, the morphism $f$ is a local complete intersection morphism (see Remark on p. 86 in [FL]). Being a finite morphism, $f$ in particular is a projective morphism. By Remark (3.5) in [Ko3], the morphism $f$ then is $\Gamma$-projective in the sense of section 3 in [Ko3]. Let $T_{f}^{\vee} \in K_{0}\left(\Gamma, \mathcal{O}_{N}\right)$ denote the equivariant cotangential element associated with $f$. It is defined as follows (see section 4 in [Ko3]): Let

$$
\operatorname{Spec}\left(\mathcal{O}_{N}\right) \stackrel{i}{\longrightarrow} \mathbb{A}=\operatorname{Spec}(A) \stackrel{p}{\longrightarrow} \operatorname{Spec}\left(\mathcal{O}_{K}\right)
$$

be a factorization of $f$ into a closed $\Gamma$-immersion $i$ and a smooth $\Gamma$-morphism $p$ of constant relative dimension $d$. Then $i$ is a regular embedding (see Remark on p. 86 in [FL]). Let $I$ denote the $\Gamma$-stable ideal in $A$ associated with $i$. Then $T_{f}^{\vee}$ is defined to be

$$
T_{f}^{\vee}:=\left[\Omega_{A / \mathcal{O}_{K}}^{1} \otimes_{A} A / I\right]-\left[I / I^{2}\right] \in K_{0}\left(\Gamma, \mathcal{O}_{N}\right)
$$

Lemma 5.1. We have:

$$
T_{f}^{\vee}=\left[\Omega_{\mathcal{O}_{N} / \mathcal{O}_{K}}^{1}\right] \quad \text { in } \quad K_{0}^{\prime}\left(\mathcal{O}_{N} \# \Gamma\right)
$$

Proof. We have a natural exact sequence

$$
I / I^{2} \rightarrow \Omega_{A / \mathcal{O}_{K}}^{1} \otimes_{A} A / I \stackrel{\epsilon}{\rightarrow} \Omega_{\mathcal{O}_{N} / \mathcal{O}_{K}}^{1} \rightarrow 0
$$

of $\Gamma$-modules over $\mathcal{O}_{N}$ (e. g. see Theorem 58i) on p. 187 in [Ma]). Being a submodule of $\Omega_{A / \mathcal{O}_{K}}^{1} \otimes A / I$, the kernel of $\epsilon$ has no torsion; thus, it is $\mathcal{O}_{N}$-projective. Furthermore, we obviously have $\operatorname{rank}_{\mathcal{O}_{N}}(\operatorname{ker}(\epsilon))=d=\operatorname{rank}_{\mathcal{O}_{N}}\left(I / I^{2}\right)$. Thus, the canonical surjection $I / I^{2} \rightarrow \operatorname{ker}(\epsilon)$ is an isomorphism. This implies that the left homomorphism in the sequence above is injective which proves Lemma 5.1.

Let $\mathfrak{D}$ denote the different associated with $N / K$.

Corollary 5.2. We have:

$$
T_{f}^{\vee}=\left[\mathfrak{D}^{-1}\right]-\left[\mathcal{O}_{N}\right] \quad \text { in } \quad K_{0}\left(\Gamma, \mathcal{O}_{N}\right)
$$

Proof. This immediately follows from Lemma 5.1 and Corollary 4.2 since $K_{0}\left(\Gamma, \mathcal{O}_{N}\right) \cong$ $K_{0}^{\prime}\left(\mathcal{O}_{N} \# \Gamma\right)$ (see Notations).

Now, we fix a $k \in \mathbb{N}$. Let $\hat{K}_{0}\left(\Gamma, \mathcal{O}_{K}\right)\left[k^{-1}\right]$ denote the $J$-adic completion of $K_{0}\left(\Gamma, \mathcal{O}_{K}\right)\left[k^{-1}\right]$ where $J:=\operatorname{ker}\left(\operatorname{rank}: K_{0}\left(\Gamma, \mathcal{O}_{K}\right) \rightarrow \mathbb{Z}\right)$ is the augmentation ideal.

Proposition 5.3. The Bott element $\theta^{k}\left(\mathfrak{D}^{-1}\right):=\sum_{i=0}^{k-1}\left[\mathfrak{D}^{-1}\right]$ is invertible in

$$
K_{0}\left(\Gamma, \mathcal{O}_{N}\right) \otimes_{K_{0}\left(\Gamma, \mathcal{O}_{K}\right)} \hat{K}_{0}\left(\Gamma, \mathcal{O}_{K}\right)\left[k^{-1}\right]
$$

and we have:

$$
\theta^{k}\left(T_{f}^{\vee}\right)^{-1}=k \cdot \theta^{k}\left(\mathfrak{D}^{-1}\right)^{-1}
$$


(see section 4 in [Ko3] for the definition of $\theta^{k}\left(T_{f}^{\vee}\right)^{-1}$ ).

Proof. For any f. g. $\mathcal{O}_{N}$-projective $\mathcal{O}_{N} \# \Gamma$-module $P$, let $\theta^{k}(P) \in K_{0}\left(\Gamma, \mathcal{O}_{N}\right)$ denote the $k$-th Bott element associated with $P$ (see section 4 in [Ko3]). By section 4 in [Ko3], there is a representation $T_{f}^{\vee}=[\Omega]-[\mathcal{N}]$ in $K_{0}\left(\Gamma, \mathcal{O}_{N}\right)$ such that $\theta^{k}(\Omega)$ is invertible in $K_{0}\left(\Gamma, \mathcal{O}_{N}\right) \otimes_{K_{0}\left(\Gamma, \mathcal{O}_{K}\right)} \hat{K}_{0}\left(\Gamma, \mathcal{O}_{K}\right)\left[k^{-1}\right]$. By Corollary 5.2 , we have $\theta^{k}\left(\mathfrak{D}^{-1}\right) \cdot \theta^{k}(\mathcal{N})=$ $k \cdot \theta^{k}(\Omega)$. Thus, $\theta^{k}\left(\mathfrak{D}^{-1}\right), \theta^{k}(\mathcal{N})$, and $\theta^{k}\left(T_{f}^{\vee}\right)$ are invertible and we have $\theta^{k}\left(T_{f}^{\vee}\right)^{-1}=$ $k \cdot \theta^{k}\left(\mathfrak{D}^{-1}\right)^{-1}$.

Pulling back the module structure along $\mathcal{O}_{K} \rightarrow \mathcal{O}_{N}$ defines an homomorphism

$$
f_{*}: K_{0}\left(\Gamma, \mathcal{O}_{N}\right) \rightarrow K_{0}\left(\Gamma, \mathcal{O}_{K}\right)
$$

Obviously, $f_{*}$ coincides with the push-forward homomorphism $f_{*}$ defined in section 3 of [Ko3]. The projection formula implies that $f_{*}$ induces an homomorphism

$$
\hat{f}_{*}: K_{0}\left(\Gamma, \mathcal{O}_{N}\right) \otimes_{K_{0}\left(\Gamma, \mathcal{O}_{K}\right)} \hat{K}_{0}\left(\Gamma, \mathcal{O}_{K}\right)\left[k^{-1}\right] \rightarrow \hat{K}_{0}\left(\Gamma, \mathcal{O}_{K}\right)\left[k^{-1}\right] .
$$

Let $\psi^{k}$ denote the $k$-th Adams operation on $K_{0}\left(\Gamma, \mathcal{O}_{K}\right)$ and $K_{0}\left(\Gamma, \mathcal{O}_{N}\right)$.

Theorem 5.4 (Equivariant Adams-Riemann-Roch formula applied to $f$ ). For all $x \in$ $K_{0}\left(\Gamma, \mathcal{O}_{N}\right)$, we have:

$$
\psi^{k} f_{*}(x)=\hat{f}_{*}\left(k \cdot \theta^{k}\left(\mathfrak{D}^{-1}\right)^{-1} \cdot \psi^{k}(x)\right) \quad \text { in } \quad \hat{K}_{0}\left(\Gamma, \mathcal{O}_{K}\right)\left[k^{-1}\right]
$$

Proof. Because of Proposition 5.3, this is the assertion of Theorem (4.5) in [Ko3] applied to $f$.

We are now going to reformulate the formula of Burns and Chinburg mentioned in the beginning of this section.

Lemma 5.5. Let $M$ be a f. g. $\mathcal{O}_{N}$-projective $\mathcal{O}_{N} \# \Gamma$-module of $\mathcal{O}_{N}$-rank $n$. Then we have:

(a) The $\mathcal{O}_{N} \# \Gamma$-module $M$ is isomorphic to a $\Gamma$-stable lattice in $N^{n}$. (In particular, any invertible $\mathcal{O}_{N} \# \Gamma$-module is isomorphic to a $\Gamma$-stable fractional ideal in $N$.)

(b) $M$ is a projective $\mathcal{O}_{K} \Gamma$-module.

Proof. Obviously, $M$ is a $\Gamma$-stable lattice in $M \otimes_{\mathcal{O}_{N}} N$. By Morita theory, the $N \# \Gamma$ module $M \otimes_{\mathcal{O}_{N}} N$ is isomorphic to the $N \# \Gamma$-module $N^{n}$. This shows assertion (a). Using (a), one easily shows as in $\S 3$ of chapter I in [F1] that $M$ is locally free over $\mathcal{O}_{K} \Gamma$. By Theorem 1.1 on p. 1 in [T], this proves assertion (b).

By Lemma 5.5, we have a push-forward homomorphism $f_{*}: K_{0}\left(\Gamma, \mathcal{O}_{N}\right) \rightarrow K_{0}\left(\mathcal{O}_{K} \Gamma\right)$ such that the following diagram commutes:

$$
\begin{aligned}
& K_{0}\left(\Gamma, \mathcal{O}_{N}\right) \\
& f_{*} \swarrow \quad \searrow f_{*} \\
& K_{0}\left(\mathcal{O}_{K} \Gamma\right) \quad \stackrel{c}{\longrightarrow} \quad K_{0}\left(\Gamma, \mathcal{O}_{K}\right) .
\end{aligned}
$$


Let $\operatorname{Pic}\left(\Gamma, \mathcal{O}_{N}\right)$ denote the group of invertible $\mathcal{O}_{N} \# \Gamma$-modules and $K_{0} \operatorname{Pic}\left(\Gamma, \mathcal{O}_{N}\right)$ the additive subgroup of $K_{0}\left(\Gamma, \mathcal{O}_{N}\right)$ generated by the multiplicative group $\operatorname{Pic}\left(\Gamma, \mathcal{O}_{N}\right)$. Furthermore, let $\operatorname{Ind}_{1}^{\Gamma}\left(\mathrm{Cl}\left(\mathcal{O}_{K}\right)\right)$ denote the image of the classical classgroup $\mathrm{Cl}\left(\mathcal{O}_{K}\right) \subset K_{0}\left(\mathcal{O}_{K}\right)$ under the the induction map $\operatorname{Ind}_{1}^{\Gamma}: K_{0}\left(\mathcal{O}_{K}\right) \rightarrow K_{0}\left(\mathcal{O}_{K} \Gamma\right)$.

We now assume that the prime divisors of $\operatorname{ord}(\Gamma)$ are unramified in $K$ and that $k$ is coprime to the exponent $e(\Gamma)$ of $\Gamma$. We fix a $k^{\prime} \in \mathbb{N}$ such that $k k^{\prime} \equiv 1 \bmod e(\Gamma)$.

Theorem 5.6 (Reformulation of a formula of Burns and Chinburg). We have for all $x \in K_{0} \operatorname{Pic}\left(\Gamma, \mathcal{O}_{N}\right)$ :

$$
\psi^{k} f_{*}(x)=f_{*}\left(k \cdot \sum_{i=0}^{k^{\prime}-1}\left[\mathfrak{D}^{-i k}\right] \cdot \psi^{k}(x)\right) \quad \text { in } \quad K_{0}\left(\mathcal{O}_{K} \Gamma\right) /\left(\operatorname{Ind}_{1}^{\Gamma}\left(\operatorname{Cl}\left(\mathcal{O}_{K}\right)\right) \oplus e(\Gamma) \mathbb{Z}\left[\mathcal{O}_{K} \Gamma\right]\right) .
$$

If the classical classgroup $\mathrm{Cl}\left(\mathcal{O}_{K}\right)$ is trivial and if the rank of $x$ is 0 , then this formula already holds in $K_{0}\left(\mathcal{O}_{K} \Gamma\right)$.

Proof. Let $\psi_{k^{\prime}}^{\mathrm{CNT}}$ denote the endomorphism of $\mathrm{Cl}\left(\mathcal{O}_{K} \Gamma\right)$ defined by Cassou-Noguès and Taylor (e. g. see section 3). For any $\mathfrak{A} \in \operatorname{Pic}\left(\Gamma, \mathcal{O}_{N}\right),(\mathfrak{A}):=\left[f_{*}(\mathfrak{A})\right]-\left[\mathcal{O}_{K} \Gamma\right]$ is a welldefined element of $\mathrm{Cl}\left(\mathcal{O}_{K} \Gamma\right)$ by Lemma 5.5. By Corollary 2.7 in [BC] and Lemma 5.5(a), we have:

$$
\psi_{k^{\prime}}^{\mathrm{CNT}}((\mathfrak{A}))=\sum_{i=0}^{k^{\prime}-1}\left(\mathfrak{D}^{-i k} \cdot \mathfrak{A}^{k}\right) \quad \text { in } \quad \mathrm{Cl}\left(\mathcal{O}_{K} \Gamma\right) / \operatorname{Ind}_{1}^{\Gamma}\left(\mathrm{Cl}\left(\mathcal{O}_{K}\right)\right) .
$$

Using Theorem 3.7, we obtain the equality

$$
\psi^{k}\left(\left[f_{*}(\mathfrak{A})\right]-\left[\mathcal{O}_{K} \Gamma\right]\right)=k \cdot \sum_{i=0}^{k^{\prime}-1}\left(\left[f_{*}\left(\mathfrak{D}^{-i k} \cdot \mathfrak{A}^{k}\right)\right]-\left[\mathcal{O}_{K} \Gamma\right]\right) \quad \text { in } \quad K_{0}\left(\mathcal{O}_{K} \Gamma\right) / \operatorname{Ind}_{1}^{\Gamma}\left(\mathrm{Cl}\left(\mathcal{O}_{K}\right)\right) .
$$

Hence, we have for all $x \in K_{0} \operatorname{Pic}\left(\Gamma, \mathcal{O}_{N}\right)$ in $K_{0}\left(\mathcal{O}_{K} \Gamma\right) / \operatorname{Ind}_{1}^{\Gamma}\left(\mathrm{Cl}\left(\mathcal{O}_{K}\right)\right)$ :

$\psi^{k} f_{*}(x)=f_{*}\left(k \cdot \sum_{i=0}^{k^{\prime}-1}\left[\mathfrak{D}^{-i k}\right] \cdot \psi^{k}(x)\right)-k k^{\prime} \cdot \operatorname{rank}_{\mathcal{O}_{N}}(x) \cdot\left[\mathcal{O}_{K} \Gamma\right]+\operatorname{rank}_{\mathcal{O}_{N}}(x) \cdot \psi^{k}\left(\left[\mathcal{O}_{K} \Gamma\right]\right)$.

Now, Theorem 1.6(e) shows Theorem 5.6.

Using an idea of the proof of Theorem 2 in $\S 4$ in [CEPT], we show in the following lemma that the right side of the formula in Theorem 5.4 coincides with the right side of the formula in Theorem 5.6 for elements of the form $x=T_{f}^{\vee} \cdot y$ with $y \in K_{0}\left(\Gamma, \mathcal{O}_{N}\right)$.

Lemma 5.7. We assume that the $\mathcal{O}_{N} \# \Gamma$-modules $\mathfrak{D}^{\operatorname{ord}(\Gamma)}$ and $\mathcal{O}_{N}$ are isomorphic. (This certainly holds if the classical classgroup $\mathrm{Cl}\left(\mathcal{O}_{K}\right)$ is trivial.) Then we have for all $y \in$ $K_{0}\left(\Gamma, \mathcal{O}_{N}\right)$ :

$\theta^{k}\left(\mathfrak{D}^{-1}\right)^{-1} \cdot \psi^{k}\left(T_{f}^{\vee} \cdot y\right)=\sum_{i=0}^{k^{\prime}-1}\left[\mathfrak{D}^{-i k}\right] \cdot \psi^{k}\left(T_{f}^{\vee} \cdot y\right) \quad$ in $\quad K_{0}\left(\Gamma, \mathcal{O}_{N}\right) \otimes_{K_{0}\left(\Gamma, \mathcal{O}_{K}\right)} \hat{K}_{0}\left(\Gamma, \mathcal{O}_{K}\right)\left[k^{-1}\right]$. 
Proof. We have:

$$
\begin{aligned}
& \theta^{k}\left(\mathfrak{D}^{-1}\right)^{-1} \cdot \psi^{k}\left(T_{f}^{\vee} \cdot y\right)= \\
& =\left(\sum_{i=0}^{k-1}\left[\mathfrak{D}^{-i}\right]\right)^{-1} \cdot\left(\left[\mathfrak{D}^{-k}\right]-\left[\mathcal{O}_{N}\right]\right) \cdot \psi^{k}(y) \quad(\text { by Corollary } 5.2) \\
& =\left(\left[\mathfrak{D}^{-1}\right]-\left[\mathcal{O}_{N}\right]\right) \cdot \psi^{k}(y) \quad \text { (geometric series) } \\
& =\left(\left[\mathfrak{D}^{-k k^{\prime}}\right]-\left[\mathcal{O}_{N}\right]\right) \cdot \psi^{k}(y) \quad\left(\text { since } \mathfrak{D}^{\operatorname{ord}(\Gamma)} \cong \mathcal{O}_{N} \text { by assumption }\right) \\
& =\left(\sum_{i=0}^{k^{\prime}-1}\left[\mathfrak{D}^{-i k}\right]\right) \cdot\left(\left[\mathfrak{D}^{-k}\right]-\left[\mathcal{O}_{N}\right]\right) \cdot \psi^{k}(y) \quad \text { (geometric series) } \\
& =\sum_{i=0}^{k^{\prime}-1}\left[\mathfrak{D}^{-i k}\right] \cdot \psi^{k}\left(T_{f}^{\vee} \cdot y\right) \quad(\text { by Corollary } 5.2) .
\end{aligned}
$$

Corollary 5.8. We assume that $\mathrm{Cl}\left(\mathcal{O}_{K}\right)$ is trivial. Then the element $f_{*}\left(T_{f}^{\vee}\right)$ in $K_{0}\left(\mathcal{O}_{K} \Gamma\right)$ or $K_{0}\left(\Gamma, \mathcal{O}_{K}\right)$ is an eigenvector of the Adams operation $\psi^{k}$ with eigenvalue $k$.

Proof. This follows from Theorem 5.6 and the proof of Lemma 5.7.

In the following closing remark, we compare Theorems 5.4 and 5.6 and point out their respective advantages.

Remark 5.9. On the one hand, Theorem 5.4 is a special case of a general (geometric) equivariant Adams-Riemann-Roch formula which can be applied even in the non-tame case, which does not need the assumption " $\operatorname{gcd}(k, \operatorname{ord}(\Gamma))=1$ ", which holds for an arbritrary $x \in K_{0}\left(\Gamma, \mathcal{O}_{N}\right)$, and which holds without passing to residues modulo $\operatorname{Ind}_{1}^{\Gamma}\left(K_{0}\left(\mathcal{O}_{K}\right)\right)$. On the other hand, Theorem 5.6 holds already for $K_{0}\left(\mathcal{O}_{K} \Gamma\right)$ without passing to $K_{0}\left(\Gamma, \mathcal{O}_{K}\right)$ or even $\hat{K}_{0}\left(\Gamma, \mathcal{O}_{K}\right)\left[k^{-1}\right]$. In particular, the multiplier $\sum_{i=0}^{k^{\prime}-1}\left[\mathfrak{D}^{-i k}\right]$ in Theorem 5.6 is definable already in $K_{0}\left(\Gamma, \mathcal{O}_{N}\right)$. Actually, Theorem 5.6 is not a reformulation but a weakening of the formula of Burns and Chinburg since we have multiplied their formula by the factor $k$ in the proof of Theorem 5.6. Finally, Theorem 5.6 consists of many formulas since there are many $k^{\prime}$ with $k k^{\prime} \equiv 1 \bmod e(\Gamma)$ (see also Remark 2.8 in $[\mathrm{BC}]$ ).

Moreover, the paper $[\mathrm{BC}]$ contains a formula for $\psi_{k}^{\mathrm{CNT}}((\mathfrak{A}))$ even if $\operatorname{gcd}(k, \operatorname{ord}(\Gamma)) \neq 1$ (see Corollary 2.4 in $[\mathrm{BC}]$ ). Since, however, there is no relation of $\psi_{k}^{\mathrm{CNT}}$ with Adams operations in this case (see Remarks 3.11 and 3.12), this formula can not be compared with the Adams-Riemann-Roch formula.

\section{References}

[AB] K. Akin and D. A. Buchsbaum, Characteristic-free representation theory of the general linear group, Adv. Math. 58 (1985), 149-200.

[ABW] D. Akin, D. A. Buchsbaum and J. Weyman, Schur functors and Schur complexes, Adv. Math. 44 (1982) 207-278. 
[B] H. BAss, "Algebraic K-theory", Math. Lecture Note Series (Benjamin, New York, 1968).

[BC] D. Burns and T. Chinburg, Adams operations and integral Hermitian-Galois representations, Amer. J. Math. 118 (1996), 925-962.

[CNT] Ph. Cassou-Noguès and M. J. Taylor, Opérations d'Adams et Groupe des classes d'Algèbre de groupe, J. Algebra 95 (1985), 125-152.

[C] T. Chinburg, Galois structure of de Rham cohomology of tame covers of schemes, Ann. of Math. 139 (1994), 443-490.

[CEPT] T. Chinburg, B. Erez, G. Pappas and M. J. Taylor, Arithmetic equivariant Riemann-Roch theorems, preprint (Manchester Centre for Pure Mathematics, number 1993/16).

[CR] C. W. Curtis and I. REIner, "Methods of representation theory with applications to finite groups and orders", vol. I, Pure Appl. Math. (Wiley, New York, 1981).

[F1] A. FröHLich, "Galois module structure of algebraic integers", Ergeb. Math. Grenzgeb. (3) $\mathbf{1}$ (Springer, New York, 1983).

[F2] A. Fröhlich, "Classgroups and Hermitian modules", Progr. Math. 48 (Birkhäuser, Boston, 1984).

[FL] W. Fulton and S. LAng, "Riemann-Roch algebra", Grundlehren Math. Wiss. 277 (Springer, New York, 1985).

[Gr1] D. R. Grayson, Higher algebraic K-theory: II, in R. M. SteIn (ed.), "Algebraic K-theory (Evanston, 1976)", Lecture Notes in Math. 551 (Springer, New York, 1976), 217-240.

[Gr2] D. R. Grayson, Localization for flat modules in algebraic K-theory, J. Algebra 61 (1979), 463-496.

[Gr3] D. R. GRAYSOn, Exterior power operations on higher $K$-theory, $K$-Theory 3 (1989), 247-260.

[Ke] M. Kervaire, Opérations d'Adams en Théorie des représentations linéaires des groupes finis, Enseign. Math. 22 (1976), 1-28.

[Ko1] B. Köck, Das Adams-Riemann-Roch-Theorem in der höheren äquivarianten K-Theorie, J. Reine Angew. Math. 421 (1991), 189-217.

[Ko2] B. Köck, Shuffle products in higher K-theory, J. Pure Appl. Algebra 92 (1994), 269-307.

[Ko3] B. KöcK, The Grothendieck-Riemann-Roch theorem in the higher $K$-theory of group scheme actions, Habilitationsschrift (Universität Karlsruhe, 1995). 
[Ko4] B. Köck, On Adams operations on the higher $K$-theory of group rings, in: G. Banaszak et al. (eds.), "Algebraic K-theory (Poznań, 1995)", Contemp. Math. 199 (Amer. Math. Soc., Providence, 1996), 139-150.

[Ko5] B. Köck, Adams operations for projective modules over group rings, to appear in Math. Proc. Cambridge Philos. Soc. 121 (1997).

[McD] I. G. Macdonald, "Symmetric functions and Hall polynomials", Oxford Math. Monographs (Clarendon Press, Oxford, 1979).

[Ma] H. Matsumura, "Commutative algebra", Math. Lecture Note Series (Benjamin, New York, 1970).

[N] J. NeukiRch, "Algebraische Zahlentheorie" (Springer, Berlin, 1992).

[Que] J. Queyrut, S-groupes des classes d'un ordre arithmétique, J. Algebra 76 (1982), 234-260.

[Q] D. QuilLen, Higher algebraic $K$-theory: I, in H. BASS (ed.), "Algebraic $K$ Theory I (Seattle, 1972)", Lecture Notes in Math. 341 (Springer, New York, 1973), 85-147.

[Se1] J. P. SerRe, "Lineare Darstellung endlicher Gruppen", Logik und Grundlagen der Mathematik (Vieweg, Braunschweig, 1972).

[Se2] J. P. Serre, "Local fields", Grad. Texts in Math. 67 (Springer, New York, 1979).

[Sw] R. G. Swan, Induced representations and projective modules, Ann. of Math. 71 (1960), 552-578.

[T] M. TAYLOR, "Classgroups of group rings", Lecture Notes Ser. 91 (Cambridge University Press, Cambridge, 1984).

Mathematisches Institut II

Universität Karlsruhe

D-76128 Karlsruhe

Germany

e-mail: Bernhard.Koeck@math.uni-karlsruhe.de 